\title{
OPTIMAL BOUNDS ON THE MODULUS OF CONTINUITY OF THE UNCENTERED HARDY-LITTLEWOOD MAXIMAL FUNCTION
}

\author{
J. M. ALDAZ, L. COLZANI AND J. PÉREZ LÁZARO
}

\begin{abstract}
We obtain sharp bounds for the modulus of continuity of the uncentered maximal function in terms of the modulus of continuity of the given function, via integral formulas. Some of the results deduced from these formulas are the following: The best constants for Lipschitz and Hölder functions on proper subintervals of $\mathbb{R} \operatorname{are~} \operatorname{Lip}_{\alpha}(M f) \leq(1+\alpha)^{-1} \operatorname{Lip}_{\alpha}(f)$, $\alpha \in(0,1]$. On $\mathbb{R}$, the best bound for Lipschitz functions is $\operatorname{Lip}(M f) \leq(\sqrt{2}-1) \operatorname{Lip}(f)$. In higher dimensions, we determine the asymptotic behavior, as $d \rightarrow \infty$, of the norm of the maximal operator associated to cross-polytopes, euclidean balls and cubes, that is, $\ell_{p}$ balls for $p=1,2, \infty$. We do this for arbitrary moduli of continuity. In the specific case of Lipschitz and Hölder functions, the operator norm of the maximal operator is uniformly bounded by $2^{-\alpha / q}$, where $q$ is the conjugate exponent of $p=1,2$, and as $d \rightarrow \infty$ the norms approach this bound. When $p=\infty$, best constants are the same as when $p=1$.
\end{abstract}

\section{Introduction.}

The constants appearing in the weak and strong type inequalities satisfied by the HardyLittlewood maximal operator, in its different variants, have been subject to considerable scrutiny. We mention, for instance, [CF], Al1], [Me1], [Me2], GMM], GM], GK], [BD], CLM], St1], St2], [St3], Bou1, [Bou2], [Bou3], Ca], [Mu, StSt, [Al2, [Al3], AlPe4], AlPe5], NaTa, and the references contained therein. Interest lies not only in determining sharp inequalities, which in general are hard to come by (in fact, no best constants are known for dimensions larger than one) but also in finding out how constants change as certain parameters (for instance, the dimension) vary, or when the type of set one is averaging over is modified, or the space of functions one is considering is changed.

Here we study the issue of optimal inequalities satisfied by the uncentered maximal operator $M$, and also its asymptotic behavior, but from a different viewpoint: Instead of considering weak and strong type inequalities, we analyze the properties of $M$ in connection with the modulus of continuity of a function. The overall emerging pattern reveals that the uncentered maximal operator improves regularity, by preserving moduli and reducing constants. But in general there is no "qualitative" improvement in the modulus. For instance, it may happen

2000 Mathematical Subject Classification. 42B25, $26 \mathrm{~A} 84$.

The first and third authors were partially supported by Grant MTM2009-12740-C03-03 of the D.G.I. of Spain.

This research was initiated during a visit of the first named author to the Università degli Studi di MilanoBicocca, and continued during a visit by the third named author to the same university; both thank the Dipartimento di Matematica e Applicazioni for its hospitality. 
that there is no change in the Hölder exponent of a function, not even in a weak, almost everywhere sense. More precisely, we will see that there are Hölder functions $f$ with exponent $\alpha \in(0,1)$, and no better than $\alpha$ on a set of positive measure, such that $M f$ is also no better than Hölder $(\alpha)$ on a set of the same measure, cf. Example 4.8. We note that the preservation of regularity does not extend to $C^{1}$ functions, see Remark 4.7.

This article is part of a wider project, which attempts to find out under which conditions and to what extent the Hardy-Littlewood maximal operator improves the regularity of functions, in different settings. If there is such an improvement, then one can try to prove variants of inequalities involving derivatives (for example, Gagliardo-Niremberg-Sobolev type inequalities) with $D M f$ replacing $D f$. In applications one often needs to consider functions more general than those belonging to Sobolev spaces, so it is natural to look for this kind of inequalities under as little regularity as possible. It is also possible to consider other maximal operators, associated to smoother approximations of the identity, but we do not pursue this line of research here.

The study of the Hardy-Littlewood maximal operator acting on spaces that measure smoothness was initiated in [Ki] by J. Kinnunen, who proved its boundedness on $W^{1, p}\left(\mathbb{R}^{d}\right)$ for $1<p \leq \infty$, and also on the Lipschitz and Hölder classes (without increasing the corresponding constants); see also [KiLi], [HaOn], [KiSa], [Ta], [Lu]. In [AlPe], part of the project outlined in the previous paragraph is carried out for functions of bounded variation and $d=1$ (the situation when $d>1$ is still not well understood, cf. [AlPe2 and [AlPe3]). Unlike the Hölder case, here a qualitative gain in regularity does occur (cf. Theorem 2.5): If $f$ is of bounded variation, then the uncentered Hardy-Littlewood maximal function $M f$ is absolutely continuous (however, the centered maximal function need not even be continuous), and furthermore, the variation is not increased by $M$. As application, a Landau type inequality under less regularity is presented in Theorem 5.1 of $[\mathrm{AlPe}$.

Given $\left(\mathbb{R}^{d},\|\cdot\|\right)$, where $\|\cdot\|$ is an arbitrary norm in $\mathbb{R}^{d}$, and $f: \mathbb{R}^{d} \rightarrow \mathbb{R}$, we present optimal integral formulas for the modulus of continuity of $M f$ in terms of the modulus of $f$. Note that distances appearing in the moduli of continuity and balls defining the maximal function, are determined according to $\|\cdot\|$. However, we always use the same underlying $d$ dimensional Lebesgue measure, regardless of the norm under consideration, i. e., there are no different normalizations of the measure for different norms. We take the viewpoint that there is (essentially) only one norm in dimension one, the usual absolute value, and this fixes Lebesgue measure in every dimension by the requirement that a cube of sidelength one has measure one. This also forces us to utilize the standard definition (via the euclidean length) and the standard normalizations, when dealing with Hausdorff measures. Such a convention (or some analogous consistency condition) is needed, for instance, to use Fubini's Theorem, and more generally, the coarea formula.

A very brief exposition of the contents of this paper follows next. The main integral formulas of the paper, valid for an arbitrary norm in the global case, where the domain under consideration is the whole space $\mathbb{R}^{d}$, appear in Theorem 2.7 and Corollary 2.14. These formulas are then specialized to the following three norms and their associated maximal functions: The $\ell_{\infty}$ norm (cf. Theorem 3.1), the $\ell_{2}$ norm (cf. Theorem 3.14), and the $\ell_{1}$ 
norm (cf. Theorem [3.16). Their associated maximal functions are respectively defined by averaging over cubes, euclidean balls, and cross-polytopes. A few consequences of these Theorems are the following: The norm of the maximal operator acting on Lipschitz functions is $\|M\|_{\mathrm{Op}(1)}=\sqrt{2}-1$ for $d=1$, cf. Corollary [3.6. For the $\ell_{\infty}$ norm the best constants in dimensions 2 and 3 are approximately 0.574 and 0.66155 . The exact values appear in Corollaries 3.7 and 3.10. For arbitrary $d,\|M\|_{\mathrm{Op}(1)}>(d-1) /(d+1)$, and the error in this estimate is $o(1 /(d+1))$, cf. Corollary 3.4. For the $\ell_{1}$ norm all constants are exactly the same as for the $\ell_{\infty}$ norm, see Theorem 3.17. By way of contrast, we note that in the euclidean case and on the class of Lipschitz functions, $\|M\|_{\mathrm{Op}(1)} \leq 2^{-1 / 2}$ in every dimension, and this bound is optimal. With respect to the preceding results, some open questions are mentioned; we indicate one now: Since $\|M f\|_{\infty}=\|f\|_{\infty}$ and (under the euclidean norm) $\|D M f\|_{\infty} \leq 2^{-1 / 2}\|D f\|_{\infty}$, the maximal operator is a contraction on $W^{1, \infty}\left(\mathbb{R}^{d}\right)$, the Sobolev space of essentially bounded functions with essentially bounded derivatives (note that the contraction is not strict, consider for instance the constant functions). Is there some analogous result for $p<\infty$ sufficiently large?

The last section of this paper deals with the maximal operator on proper subintervals of $\mathbb{R}$. In this case, the operator norm of $M$ when acting on the Hölder and Lipschitz classes is $\|M\|_{\mathrm{Op}(\alpha)}=(1+\alpha)^{-1}$ (cf. Corollary 4.5). The local, higher dimensional case is not studied here; we only point out that constants when $d>1$ depend on the geometry of the domain, as was to be expected, cf. Remark 4.9.

Finally, we mention that the centered maximal operator $M^{c}$ associated to euclidean balls satisfies $\left\|M^{c}\right\|_{\mathrm{Op}(\alpha)}=1$ in all dimensions, so Lipschitz and Hölder constants are in general not reduced (cf. Remark 2.4). Thus, the preceding results, together with Theorem 2.5 of $\mathrm{AlPe}$ mentioned above, suggest that from the viewpoint of regularity the uncentered maximal operator is a more natural object of study than the centered one.

\section{DeFinitions AND GLOBAL RESUlts FOR ARBITRARY NORMS.}

Definition 2.1. Let $U \subset \mathbb{R}^{d}$ be an open set, let $\|\cdot\|$ be a norm on $\mathbb{R}^{d}$, and let $B$ be a generic ball with respect to this norm. Given a locally integrable function $f: U \rightarrow \mathbb{R}$, the noncentered Hardy-Littlewood maximal function $M f$ is defined by

$$
M f(x):=\sup _{x \in B \subset U} \frac{1}{|B|} \int_{B}|f(y)| d y .
$$

Here $|B|$ stands for the Lebesgue measure of $B$. Regarding the centered maximal function $M^{c} f(x)$, one requires that balls be centered at $x$ rather than just containing it, but everything else is as in the uncentered case.

Definition 2.2. The modulus of continuity of a function $f$ is

$$
\omega(f, \delta):=\sup \{|f(x)-f(y)|:\|x-y\| \leq \delta\} .
$$

We point out that both definitions depend on the norm under consideration (while the underlying Lebesgue measure does not). Although maximal functions and moduli of continuity 
obtained via different norms will always be pointwise comparable, from the viewpoint of best constants distinctions cannot be neglected. Indeed, when the euclidean $\left(\ell_{2}\right)$ and the max $\left(\ell_{\infty}\right)$ norms are used in $\mathbb{R}^{d}, d>1$ the results vary. Somewhat surprisingly, since already in dimension 3 cubes and cross-polytopes are very different geometrical objects, best constants associated to the $\ell_{\infty}$ norm and arbitrary moduli of continuity are exactly the same as those obtained under the $\ell_{1}$ norm. Let us emphasize, though, that our results say nothing, for instance, about the maximal function associated to cubes when the length used is the euclidean distance. For us, fixing a norm fixes the balls we average over; once we select, say, the euclidean norm, the maximal function considered in our theorems will be the one associated to euclidean balls.

The following theorem is due to Juha Kinnunen, cf. [Ki]. While not explicitly stated there, its proof appears in [Ki], pp. 120-121, Remark 2.2 (iii). A small variant of the argument yields the boundedness in $W^{1, p}(\mathbb{R}), 1<p \leq \infty$, of the maximal operator (Remark 2.2 (i) of [Ki]); an abstract version can be found in Theorem 1 of [HaOn], where it is applied to the spherical maximal operator.

In the next theorem $\mathcal{M}$ denotes a generic Hardy-Littlewood maximal operator (it could be centered or noncentered, associated to euclidean or to other balls).

Theorem 2.3. (J. Kinnunen). Let $f: \mathbb{R}^{d} \rightarrow \mathbb{R}$ be locally integrable. Then, for every $\delta>0$,

$$
\omega(\mathcal{M} f, \delta) \leq \omega(|f|, \delta) .
$$

Proof. Let $h, x \in \mathbb{R}^{d}$ and set $f_{h}(x):=f(x+h)$. By commutativity of $\mathcal{M}$ with translations, $\mathcal{M} f(x+h)=\mathcal{M} f_{h}(x)$, and by subadditivity, $\left|\mathcal{M} f_{h}-\mathcal{M} f\right| \leq \mathcal{M}\left(\left|f_{h}\right|-|f|\right)$. Since averages never exceed a supremum, we have $\sup _{x \in \mathbb{R}^{d}} \mathcal{M}\left(\left|f_{h}\right|-|f|\right)(x) \leq \sup _{x \in \mathbb{R}^{d}}\left(|| f_{h}|-| f||\right)(x)$, and now (2.3.1) follows by taking the sup over $\|h\| \leq \delta$.

Remark 2.4. This simple result already contains the sharp bound for the centered maximal operator $M^{c}$ on the Lipschitz and Hölder classes. Let $\psi(x)=\max \{1-|x|, 0\}$ be defined on $\mathbb{R}$; clearly, $M^{c} \psi(x)=\psi(x)$ for every $x \in[-1 / 2,1 / 2]$, so both functions have the same Lipschitz constant: $\operatorname{Lip}\left(M^{c} \psi\right)=\operatorname{Lip}(\psi)=1$. Now, this example can be easily adapted to higher dimensions (for instance, by making the corresponding function depend only on the first coordinate), and it follows that the centered maximal operator on $\mathbb{R}^{d}$ does not in general reduce the Lipschitz constant of a function. By way of contrast, we mention that the uncentered maximal operator on $\mathbb{R}$ satisfies $\operatorname{Lip}(M f) \leq(\sqrt{2}-1) \operatorname{Lip}(f)$, cf. Corollary 3.6 below, while on $\mathbb{R}^{d}$ with the Euclidean norm, the bound $\operatorname{Lip}(M f) \leq 2^{-1 / 2} \operatorname{Lip}(f)$ holds uniformly in the dimension (Theorem 3.14).

As for Hölder functions, the preceding example can be easily modified to yield the same conclusion. In one dimension, set $\eta(x):=\max \left\{1-|x|^{\alpha}, 0\right\}$ when $x \leq 0$, set $\eta(x):=1+|x|^{\alpha}$ on $[0,1 / 4]$, and finally, extend $\eta$ to $[1 / 4, \infty)$ by reflection about the $x=1 / 4$ axis. Then, by concavity of $\eta$ on $0<x<1 / 4, M^{c} \eta(x)=\eta(x)$ for every $x \in[0,1 / 4]$, and thus both functions have the same Hölder constant (we mention that the uncentered operator improves Hölder constants, see formula (3.3.1) below). It is clear that this example can also be adapted to higher dimensions. 
Our aim in this paper is to find best inequalities in the spirit of (2.3.1) for the uncentered maximal operator, which, as noted in the introduction, has better properties regarding the regularization of functions than its centered relative.

Definition 2.5. A function $\omega:[0, \infty) \rightarrow[0, \infty)$ is a modulus of continuity if it is the modulus of continuity of a uniformly continuous function, i.e., if there is a uniformly continuous function $f$ such that for all $\delta \geq 0, \omega(\delta)=\omega(f, \delta)$.

Remark 2.6. Often a modulus of continuity $\omega:[0, \infty) \rightarrow[0, \infty)$ is defined as a continuous, nondecreasing, subadditive function (so $\omega(a+b) \leq \omega(a)+\omega(b)$ ), vanishing at zero. Note that from continuity and subadditivity it follows that $\omega$ is uniformly continuous. It is well known and not difficult to check that the modulus of continuity of a uniformly continuous function $f$ has these properties, while given any $\omega$ satisfying the above conditions, there is a uniformly continuous $f$ such that $\omega(\cdot)=\omega(f, \cdot)$, namely $\omega$ itself. We do not assume that moduli of continuity are bounded, so our results apply to general Lipschitz and Hölder functions. To avoid trivialities, given a generic modulus of continuity, we shall assume it is not identically zero.

The next theorem, and its corollary 2.14, contain the main integral formulas of the paper, valid (in the global case) for all norms and all dimensions. Note that on $\mathbb{R}^{d}$ the value of $M f(x)$ is the same regardless of whether the balls we average over are taken to be open or closed. We shall assume whatever is more convenient at any given point. For instance, in the next theorem and its proof we suppose that balls are closed. Additionally, we use the following notation: $a_{n} \uparrow b$ (resp. $a_{n} \downarrow b$ ) means that the sequence $\left\{a_{n}\right\}$ converges to $b$ in a monotone increasing (resp. decreasing) fashion.

Theorem 2.7. Let $d \geq 1$ and let $f$ be a locally integrable function on $\mathbb{R}^{d}$. Then, for every norm $\|\cdot\|$ on $\mathbb{R}^{d}$ and every $t \geq 0$,

$$
\omega(M f, t) \leq \sup _{\left\{v \in \mathbb{R}^{d}:\|v\|=1\right\}} \inf _{\left\{c \in \mathbb{R}^{d}, R>0:\|v-c\| \leq R\right\}} \frac{1}{|B(0,1)|} \int_{B(0,1)} \omega(|f|, t\|c+R u\|) d u .
$$

The preceding inequality is optimal in the sense that given a modulus of continuity $\omega$, there exists a function $\psi$ such that letting $f=\psi$ in 2.7.1), the following equalities hold: For all $t>0$, we have $\omega(\psi, t)=\omega(t)$, and furthermore,

$$
\omega(M \psi, t)=\sup _{\left\{v \in \mathbb{R}^{d}:\|v\|=1\right\}} \inf _{\left\{c \in \mathbb{R}^{d}, R>0:\|v-c\| \leq R\right\}} \frac{1}{|B(0,1)|} \int_{B(0,1)} \omega(t\|c+R u\|) d u .
$$

If $v$ is a unit vector, to find its associated infimum in (2.7.2) it is enough to consider the set

$$
\left\{c \in \mathbb{R}^{d}, R>0:\|v-c\|=R \text { and } R \leq 1\right\} .
$$

As for the extremal functions $\psi$ appearing in (2.7.2), they have the following form: If $\omega$ is bounded, we can take $\psi(x)=\|\omega\|_{L^{\infty}([0, \infty))}-\omega(\|x\|)$, while if $\omega$ is unbounded, we set $\psi(x)=\sum_{n=1}^{\infty}\left(\Omega_{n}-w\left(\left\|x-a_{n}\right\|\right)\right)^{+}$, where $\left\{\Omega_{n}\right\}_{1}^{\infty} \uparrow \infty$ and $\left\{a_{n}\right\}_{1}^{\infty}$ diverges to infinity so fast, 
that on the support of each spike the maximal function does not depend on any of the other spikes.

Observe that (2.7.1) is stronger, for the uncentered maximal operator, than Kinnunen's inequality $\omega(M f, t) \leq \omega(|f|, t)$, as can be seen by letting $R \rightarrow 0$ (and hence $c \rightarrow v$ ). Note also that the right hand side of (2.7.2) depends only on the modulus of continuity and on the norm. Regarding the extremal functions, our strategy to find them is easy to explain (and the proof of the theorem shows that it works): Suppose $\omega$ is a bounded modulus of continuity, and suppose that a given function $g$ has a global maximum at $x$. Then $M g(x)=g(x)$, and in order to maximize $M g(x)-M g(y)$ for each $y \neq x$, we want to minimize $M g(y)$. Thus $g$ should have the fastest possible decay allowed by $\omega$ in every direction (and hence the least possible mass). This is precisely what $\psi(x):=\|\omega\|_{L^{\infty}([0, \infty))}-\omega(\|x\|)$ does. A similar observation (in a local sense) can be made for unbounded moduli. Finally, we mention that while it is natural to suspect that the centers $c$ in (2.7.3) associated to $v$ should be chosen so that $B(c, R) \subset B(0,1)$, Theorem 2.7 does not make any such assertion, and in fact, it may be false in general. But we shall see in the next section that it is indeed true for the $\ell_{1}, \ell_{2}$ and $\ell_{\infty}$ norms.

Proof. Given $f \geq 0$, we assume that the associated modulus of continuity $\omega(f, t)$ is finite for every $t>0$, for otherwise there is nothing to prove. Hence, we take $f$ to be locally bounded. We may also assume that $M f$ is not constant, and in particular, that it is not identically $\infty$. Next, choose $x, y \in \mathbb{R}$ and suppose that $M f(y)<M f(x)$. Set

$$
E:=\left\{b \in \mathbb{R}^{d}, T>0:\|(y-x)-b\| \leq T\right\} \quad \text { and } \quad F:=\left\{a \in \mathbb{R}^{d}, S>0:\|x-a\| \leq S\right\} .
$$

Then, for all $(a, S) \in F$ we have $E \subset\left\{b \in \mathbb{R}^{d}, T>0: y \in B(a+b, S+T)\right\}$ by the triangle inequality. Hence

$$
\begin{gathered}
M f(x)-M f(y)=\sup _{F} \frac{1}{|B(a, S)|} \int_{B(a, S)} f(u) d u-M f(y) \\
\leq \sup _{F} \inf _{E}\left(\frac{1}{|B(a, S)|} \int_{B(a, S)} f(u) d u-\frac{1}{|B(a+b, S+T)|} \int_{B(a+b, S+T)} f(u) d u\right) \\
\leq \sup _{F} \inf _{E}\left(\frac{1}{|B(0,1)|} \int_{B(0,1)}|f(a+S u)-f(a+b+(S+T) u)| d u\right) \\
\leq \sup _{F} \inf _{E} \frac{1}{|B(0,1)|} \int_{B(0,1)} \omega(f,\|b+T u\|) d u \\
=\inf _{E} \frac{1}{|B(0,1)|} \int_{B(0,1)} \omega(f,\|b+T u\|) d u,
\end{gathered}
$$

where the $\sup _{F}$ has been deleted from the last line since neither $a$ nor $S$ appear in the integral. By symmetry of $B(0,1)$, the inequality

$$
|M f(x)-M f(y)| \leq \inf _{E} \frac{1}{|B(0,1)|} \int_{B(0,1)} \omega(f,\|b+T u\|) d u
$$


also holds when $M f(y)>M f(x)$, and thus it always holds. Writing in (2.7.4) $c\|y-x\|=b$, $R\|y-x\|=T$, and $y-x=\|y-x\| v$, where $\|v\|=1$, we have

$$
|M f(x)-M f(y)| \leq \inf _{\left\{c \in \mathbb{R}^{d}, R>0:\|v-c\| \leq R\right\}} \frac{1}{|B(0,1)|} \int_{B(0,1)} \omega(f,\|y-x\|\|c+R u\|) d u .
$$

Since the right hand side of the last inequality is increasing in $\|y-x\|$, it follows that for every $t>0$,

$$
\begin{aligned}
& \omega(M f, t)=\sup _{\left\{x, y \in \mathbb{R}^{d}:\|x-y\| \leq t\right\}}|M f(x)-M f(y)| \\
& \leq \sup _{\left\{v \in \mathbb{R}^{d}:\|v\|=1\right\}} \inf _{\left\{c \in \mathbb{R}^{d}, R>0:\|v-c\| \leq R\right\}} \frac{1}{|B(0,1)|} \int_{B(0,1)} \omega(f, t\|c+R u\|) d u .
\end{aligned}
$$

Next we prove that this inequality is sharp. Suppose first that $\omega$ is a bounded modulus of continuity. Set $\Omega:=\|\omega\|_{L^{\infty}([0, \infty))}$, and write $\psi(x):=\Omega-\omega(\|x\|)$. Fix $t>0$. If $x$ satisfies $0<\|x\| \leq t$, then we can express $x=t v$, where $0<\|v\| \leq 1$, and we get

$$
\omega(M \psi, t) \geq M \psi(0)-M \psi(x)=\inf _{\left\{c \in \mathbb{R}^{d}, R>0:\|v-c\| \leq R\right\}} \frac{1}{|B(0,1)|} \int_{B(0,1)} \omega(t\|c+R u\|) d u .
$$

Now the result follows by taking the supremum over all $v$ such that $0<\|v\| \leq 1$. If $\omega$ is unbounded, we modify $\psi$ it as follows: Take a sequence of suitably chosen "spike" functions $\left(\Omega_{n}-\omega\left(\left\|x-a_{n}\right\|\right)\right)^{+}$, and then set $\psi(x)=\sum_{n=1}^{\infty}\left(\Omega_{n}-w\left(\left\|x-a_{n}\right\|\right)\right)^{+}$. "Suitably chosen" in the preceding sentence means that the heights $\Omega_{n}$ tend to infinity and the different spikes are placed so far apart (i.e., $a_{n} \rightarrow \infty$ so fast) that on the support of each spike, the others need not be taken into account when computing $M \psi(x)$ (in particular, different spikes will have disjoint supports, so there are no convergence issues with the series defining $\psi$ ).

In order to apply formula (2.7.5), it is useful to narrow down as much as possible where the infimum occurs. First we show that it is enough to consider $R \leq 1$. Fix a unit vector $v$ and suppose $R>1$. Since $\|v-0\|=1 \leq R$, the origin is an admissible center $c$ associated to $v$. But then averaging $\omega(f, t\|\cdot\|)$ over $B(0,1)$ yields a value no larger than averaging over any other ball $B(c, R)$ containing $v$, for every vector in $B(c, R) \backslash B(0,1)$ has norm larger than one, hence larger than the norm of any vector in $B(0,1) \backslash B(c, R)$, and additionally $|B(c, R) \backslash B(0,1)|>|B(0,1) \backslash B(c, R)|$.

Next we prove that it is enough to consider pairs $(c, R)$ for which $\|v-c\|=R$. Suppose $\|v-c\|<R$. Since the continuous function $r \mapsto r R-\|v-r c\|$ changes sign on $[0,1]$, there exists an $r_{0} \in[0,1]$ such that $\left\|v-r_{0} c\right\|=r_{0} R$ and, for such an $r_{0}$, we have $\omega\left(f, t\left\|r_{0} c+r_{0} R u\right\|\right) \leq$ $\omega(f, t\|c+R u\|)$. Hence, we do no worse by using the pair $\left(r_{0} c, r_{0} R\right)$ instead of $(c, R)$, and (2.7.3) follows.

Remark 2.8. We mention that to obtain (2.7.3), instead of the selfcontained argument given above, we could have applied Anderson's Theorem (cf., for instance, Theorem 1.11, pg. 376 of [Ga], or Theorem 1 of $[\mathrm{An}]$ ), which for nonnegative, integrable, symmetric and unimodal 
functions $f$, and origin symmetric convex bodies $K \subset \mathbb{R}^{d}$, tells us that

$$
\int_{K} f(x+c y) d x \geq \int_{K} f(x+y) d x,
$$

where $0 \leq c \leq 1$ and $y \in \mathbb{R}^{d}$. Since we shall use Anderson's Theorem later on, it is stated here for easy reference.

Definition 2.9. Given a modulus of continuity $\omega$, define the Lipschitz space $\operatorname{Lip}(\omega)=$ $\operatorname{Lip}(\omega, X)$ via the seminorm

$$
\|f\|_{\operatorname{Lip}(\omega, X)}:=\sup _{\{x, y \in X: x \neq y\}} \frac{|f(x)-f(y)|}{\omega(\|x-y\|)}=\sup _{t>0} \frac{\omega(f, t)}{\omega(t)} .
$$

Then $f \in \operatorname{Lip}(\omega, X)$ if (and only if) $\|f\|_{\operatorname{Lip}(\omega, X)}<\infty$. In this section and the next we will always have $X=\mathbb{R}^{d}$, so reference to $X$ shall usually be omitted. Next, set

$$
\|M\|_{\mathrm{Op}(\omega)}:=\sup _{\|f\|_{\operatorname{Lip}(\omega)} \neq 0} \frac{\|M f\|_{\operatorname{Lip}(\omega)}}{\|f\|_{\operatorname{Lip}(\omega)}}=\sup _{\|f\|_{\operatorname{Lip}(\omega)}=1}\|M f\|_{\operatorname{Lip}(\omega)} .
$$

When $\omega(t)=t^{\alpha}$ and $0<\alpha \leq 1$, we use $\operatorname{Lip}_{\alpha}(X)$ (or just $\operatorname{Lip}(\alpha)$ ) to denote the corresponding spaces of Hölder continuous functions and of Lipschitz functions on $X, \operatorname{Lip}_{\alpha}(f)$ to denote $\|f\|_{\operatorname{Lip}(\omega)}$, and $\|M\|_{\mathrm{Op}(\alpha)}$ to denote $\|M\|_{\mathrm{Op}(\omega)}$. If $\alpha=1$, we often omit it, simply writing $\operatorname{Lip}(X)$ and $\operatorname{Lip}(f)$.

While the notation does not make it explicit, since we are considering functions defined on $\left(\mathbb{R}^{d},\|\cdot\|\right),\|M\|_{\mathrm{Op}(\omega)}$ depends both on $d$ and on $\|\cdot\|$. But dimension and norm will always be clear from context.

Remark 2.10. Kinnunen's Theorem (2.3) shows that $\|\mathcal{M}\|_{\mathrm{Op}(\omega)} \leq 1$ for all sorts of maximal operators $\mathcal{M}$, since for every $t>0$ and every $f$ with $\|f\|_{\operatorname{Lip}(\omega, X)} \leq 1$, we have $\omega(\mathcal{M} f, t) \leq$ $\omega(|f|, t) \leq \omega(f, t) \leq \omega(t)$.

Example 2.11. Note that for $f \in \operatorname{Lip}(\omega)$, its norm $\|f\|_{\operatorname{Lip}(\omega)}$ depends not only on the space $\operatorname{Lip}(\omega)$, but also on the modulus $\omega$ used to define it (we write norm for short, even though we mean seminorm). Consider, for instance, the functions on $\mathbb{R}$ that are both bounded and Lipschitz. As a set, this space can be defined via many moduli, for example $\omega(t):=\min \{t, 1\}$ and $\omega^{\prime}(t):=\min \{t, 1 / 2\}$. Then $\psi(x)=(1-|x|)^{+}=\max \{0,1-|x|\}$ has norm 1 in $\operatorname{Lip}(\omega)$ and norm 2 in $\operatorname{Lip}\left(\omega^{\prime}\right)$. We also mention that since $\lim _{t \rightarrow \infty} \omega(\mathcal{M} \psi, t)=1$, we have $\|M\|_{\text {Op }(\omega)}=1$, so the upper bound 1 can be attained by the uncentered maximal operator. But we shall see that for many standard moduli strict inequality holds.

Remark 2.12. Identifying some extremal functions, as we do in Theorem 2.7, allows us to immediately improve the general bound 1 in the uncentered case, on Lipschitz functions, for every dimension $d$, and all norms.

Corollary 2.13. Fix $d \geq 1$. Given any norm $\|\cdot\|$ on $\mathbb{R}^{d}$, the associated maximal operator acting on Lipschitz functions satisfies $\|M\|_{\mathrm{Op}(1)} \leq d /(d+1)$. 
Proof. Let $\psi(x)=\max \{1-\|x\|, 0\}$ (since we are in the Lipschitz case, it is immaterial which height $\Omega$ we select in Theorem 2.7, so we just pick $\Omega=1$ ). Given a vector $v$ with $\|v\|=1$, we estimate $M \psi(v)$ by integrating over the support of $\psi$, that is, over the unit ball according to $\|\cdot\|$. This gives a lower bound for $M \psi$ on the unit sphere, and hence an upper bound for $\|M\|_{\mathrm{Op}(1)}$. Since for $d \geq 1$ the average of the cone over the unit ball is $1 /(d+1)$, we have $\|M\|_{\mathrm{Op}(1)} \leq 1-1 /(d+1)$.

Of course, the very general but otherwise rather crude estimates given by the preceding corollary cannot be expected to be sharp. In dimension one there is essentially one norm, and the best constant is $\sqrt{2}-1$, as will be seen below, rather than $1 / 2$. We shall show that in dimensions two and three the constants $2 / 3$ and $3 / 4$ can be improved when dealing with the $\ell_{1}$ and $\ell_{\infty}$ norms.

Let $f$ be a smooth, compactly supported function on $\mathbb{R}^{d}$, and recall that $\operatorname{Lip}(M f)=$ $\|D M f\|_{\infty}$. Since by the previous corollary $\|D M f\|_{\infty} \leq d /(d+1)\|D f\|_{\infty}$, it is natural to suspect that if $p_{d}$ is high enough, for every $p \geq p_{d}$ one can find a $c_{p} \in(0,1)$ such that $\|D M f\|_{p} \leq c_{p}\|D f\|_{p}$ (with $c_{p}$ independent of $f$, see also question 2 below). But in this paper we study the size of $\|D M f\|_{p}$ only when $p=\infty$.

Note also that asymptotically the above corollary does not improve the general upper bound 1. Thus, it is natural to enquire whether bounds strictly less than 1 and independent of the dimension can be obtained, by a more careful choice of averaging ball. It turns out that for cubes (with sides parallel to the axes, that is, $\ell_{\infty}$ balls) and cross-polytopes ( $\ell_{1}$ balls) the constant 1 is the correct asymptotic value, and the trivial upper bound $d /(d+1)$ from the previous result is "essentially" optimal (actually, we shall see later that the lower bound $(d-1) /(d+1)$ is a better asymptotic estimate). However, substantial improvement is possible for Euclidean $\left(\ell_{2}\right)$ balls, and thus, the same question on the $p$ norm of the derivative arises: Can we have $c_{p}<1$ for $p<\infty$ sufficiently high? And can we take $p$ to be independent of the dimension?

We conclude this section by using the notation from Definition 2.9 to summarize the main contents of Theorem 2.7. While formula (2.14.1) below does not look very promising, due to the successive appearance of two suprema and one infimum, the fact is that it will allow us to obtain optimal asymptotic estimates for the norms considered in the next section, and in some cases we will be able to actually compute the number $\|M\|_{\mathrm{Op}(\omega)}$.

Corollary 2.14. Let $\|\cdot\|$ be a norm on $\mathbb{R}^{d}$, and let $\omega$ be a modulus of continuity. Then the associated maximal operator $M$ acting on $\operatorname{Lip}(\omega)$ has norm given by

$$
\|M\|_{\mathrm{Op}(\omega)}=\sup _{t>0} \sup _{\left\{v \in \mathbb{R}^{d}:\|v\|=1\right\}} \inf _{\left\{c \in \mathbb{R}^{d}, 0<R \leq 1:\|v-c\|=R\right\}} \frac{1}{\omega(t)} \int_{B(0,1)} \omega(t\|c+R u\|) \frac{d u}{|B(0,1)|} .
$$

Proof. Using one of the extremal functions $\psi$ found in Theorem 2.7 we have $\|M\|_{\mathrm{Op}(\omega)}=$ $\|M \psi\|_{\operatorname{Lip}(\omega)}$, and now the result follows from (2.7.2) together with (2.7.3). 


\section{Bounds IN THE $\ell_{\infty}, \ell_{2}$ AND $\ell_{1}$ CASES.}

Next we specialize Theorem 2.7 and Corollary 2.14 to the three norms in the title of this section. This specialization will allow us to find explicit constants, at least for low dimensions and in the case of cubes (where the needed arguments seem to be simpler). As we mentioned, however, all constants will turn out to be exactly the same when working with the $\ell_{\infty}$ and $\ell_{1}$ norms.

Theorem 3.1. Let $M$ be the uncentered maximal operator associated to balls defined by the $\ell_{\infty}$ norm on $\mathbb{R}^{d}$, i.e., to cubes with sides parallel to the coordinate axes, and let $\omega$ be a modulus of continuity. Then

$$
\|M\|_{\mathrm{Op}(\omega)}=\sup _{t>0}\left\{\inf _{0 \leq s \leq 1} \frac{1}{\omega(t)} \int_{[-s, 1]^{d}} \omega\left(t\|x\|_{\infty}\right) \frac{d x}{(1+s)^{d}}\right\}
$$

or equivalently,

$$
\|M\|_{\mathrm{Op}(\omega)}=\sup _{t>0}\left\{\inf _{0 \leq s \leq 1} \frac{d}{(1+s)^{d} \omega(t)}\left[2^{d} \int_{0}^{s} u^{d-1} \omega(t u) d u+\int_{s}^{1}(u+s)^{d-1} \omega(t u) d u\right]\right\} .
$$

Moreover, if we choose the same modulus $\omega$ for every dimension $d$, then $\|M\|_{\mathrm{Op}(\omega)}$ is nondecreasing in $d$, and $\lim _{d \rightarrow \infty}\|M\|_{\mathrm{Op}(\omega)}=1$.

Of the two expressions for $\|M\|_{\mathrm{Op}(\omega)}$ given in the preceding theorem, (3.1.1) is the one with a clearer geometric content: It says that an optimal choice for $v$ is $(1,1, \ldots, 1)$, and the associated minimizing cube contains the origin and is contained in $[-1,1]^{d}$. On the other hand, while harder to interpret, formula (3.1.2) turns out to be computationally much more convenient.

Proof. From (2.14.1) it follows that

$$
\|M\|_{\mathrm{Op}(\omega)}=\sup _{t>0} \sup _{\left\{v \in \mathbb{R}^{d}:\|v\|_{\infty}=1\right\}} \inf _{\left\{c \in \mathbb{R}^{d}, R>0:\|v-c\|_{\infty}=R \leq 1\right\}} \frac{1}{\omega(t)} \int_{[-1,1]^{d}} \omega\left(t\|c+R u\|_{\infty}\right) \frac{d u}{2^{d}},
$$

so if we write $v_{0}:=(1,1, \ldots, 1)$, then

$$
\|M\|_{\mathrm{Op}(\omega)} \geq \sup _{t>0} \inf _{\left\{c \in \mathbb{R}^{d}, R>0:\left\|v_{0}-c\right\|_{\infty}=R \leq 1\right\}} \frac{1}{\omega(t)} \int_{[-1,1]^{d}} \omega\left(t\|c+R u\|_{\infty}\right) \frac{d u}{2^{d}} .
$$

We claim that the infimum in (3.1.4) is attained when $c=(1-R, 1-R, \ldots, 1-R)$ for some $R \in(0,1]$ (which may vary with the dimension). Let $c=\left(c_{1}, c_{2}, \ldots, c_{d}\right)$ satisfy $\left\|v_{0}-c\right\|_{\infty}=$ $R \leq 1$. Then $c_{1}, c_{2}, \ldots, c_{d} \geq 1-R$. Suppose $c_{1}>1-R$ (else, do nothing and consider $c_{2}$ instead). Translate $c$ to $c^{1}:=\left(1-R, c_{2}, \ldots, c_{d}\right)$, by moving it parallel to $e_{1}$. Now, for every $x \in B(c, R) \backslash B\left(c^{1}, R\right),\left|x_{1}\right|>1$, while for every $x \in B\left(c^{1}, R\right) \backslash B(c, R),\left|x_{1}\right| \leq 1$. Since parallel transport in the direction of $e_{1}$ does not change any of the other coordinates, the average value of $\|\cdot\|_{\infty}$ is not increased. Then repeat this process with each coordinate. 
Next, note that the infimum is attained when $1 / 2 \leq R \leq 1$. This immediately follows from the fact that for each $u \in B(0,1)$, the function $f(R):=\left\|(1-R) v_{0}+R u\right\|_{\infty}$ is decreasing on $(0,1 / 2]$, since each coordinate function $\left|1-R+R u_{i}\right|=1-\left(1-u_{i}\right) R$ is decreasing there. Thus

$$
\|M\|_{\mathrm{Op}(\omega)} \geq \sup _{t>0} \inf _{\{1 / 2 \leq R \leq 1\}} \frac{1}{\omega(t)} \int_{[-1,1]^{d}} \omega\left(t\left\|(1-R) v_{0}+R u\right\|_{\infty}\right) \frac{d u}{2^{d}} .
$$

On the other hand, using (3.1.3) and, for each each unit vector $v$, taking the infimum over a smaller set of associated centers $c$, we get

$$
\|M\|_{\mathrm{Op}(\omega)} \leq \sup _{t>0} \sup _{\left\{v \in \mathbb{R}^{d}:\|v\|_{\infty}=1\right\}} \inf _{\{1 / 2 \leq R \leq 1\}} \frac{1}{\omega(t)} \int_{[-1,1]^{d}} \omega\left(t\|(1-R) v+R u\|_{\infty}\right) \frac{d u}{2^{d}} .
$$

We show that the supremum over unit vectors is attained on $v_{0}$, so in fact the right hand sides of (3.1.5) and (3.1.6) are equal. From this, (3.1.1) follows by making the change of variable $x=(1-R) v_{0}+R u$, and relabeling $s=2 R-1$. Fix $R \in[1 / 2,1]$, and let $v=\left(v_{1}, \ldots, v_{d}\right)$ satisfy $\|v\|_{\infty}=1$. By symmetry considerations we may assume that $v_{1}, \ldots, v_{d} \geq 0$. Now we argue as before. If $v_{1}=1$ do nothing and move to $v_{2}$. Else, $v_{1}<1$, so shift $v$ to $v^{1}:=\left(1, v_{2}, \ldots, v_{d}\right)$ by parallel transport in the direction of $e_{1}$. Then for every $x \in B((1-R) v, R) \backslash B\left((1-R) v^{1}, R\right)$, $(1-R) v_{1}-R<x_{1}<0$, so $\left|x_{1}\right|<\left|(1-R) v_{1}-R\right|$, while if $x \in B\left((1-R) v^{1}, R\right) \backslash B((1-R) v, R)$, we have $\left|x_{1}\right|>(1-R) v_{1}+R$. Since the average value of $\left|x_{1}\right|$ increases after the shift and the other coordinates do not change, the average value of $\|\cdot\|_{\infty}$ increases. Then repeat this process with each coordinate.

To obtain (3.1.2) from (3.1.1), break up the integral appearing in (3.1.1) into the regions $\left\{\|x\|_{\infty}<s\right\}$ and $\left\{\|x\|_{\infty} \geq s\right\}$, and then separate these into the sets where $\left|x_{i}\right|=\|x\|_{\infty}$, for $i=1, \ldots, d$. Note, for instance, that if we are working over $\left\{\|x\|_{\infty} \geq s\right\} \cap\left\{x_{1}=\|x\|_{\infty}\right\}$, then, for a fixed value of $x_{1}$, the coordinates $x_{2}, \ldots, x_{d}$ of the associated vertical section $E_{x_{1}}$ satisfy $-s \leq x_{i} \leq x_{1}$, so $\left|E_{x_{1}}\right|=\left(s+x_{1}\right)^{d-1}$. A similar remark can be made about the integral over $\left\{\|x\|_{\infty}<s\right\}$, so applying Fubini's Theorem we obtain (3.1.2).

Next we show that the norm of $M$ does not decrease when the dimension changes from $d$ to $d+1$ if we keep the same modulus $\omega$. For notational simplicity, we suppose that $d=1$. The argument for arbitrary $d$ is the same. Let $s_{d} \in[0,1]$ be the minimizing value of $s$ in dimension $d$. Then

$$
\begin{gathered}
\frac{1}{\left(1+s_{2}\right)^{2}} \int_{\left[-s_{2}, 1\right]^{2}} \omega(t \max \{|x|,|y|\}) d x d y \geq \frac{1}{\left(1+s_{2}\right)^{2}} \int_{-s_{2}}^{1} \int_{-s_{2}}^{1} \omega(t|x|) d x d y \\
=\frac{1}{1+s_{2}} \int_{-s_{2}}^{1} \omega(t|x|) d x \geq \frac{1}{1+s_{1}} \int_{-s_{1}}^{1} \omega(t|x|) d x
\end{gathered}
$$

To finish, we show that as the dimension $d \rightarrow \infty$, for a fixed modulus $\omega$ we have $\|M\|_{\mathrm{Op}(\omega)} \rightarrow$ 1. This is simply a consequence of the fact that for every $s \in[0,1]$, in high dimensions the measure of the cube $[-s, 1]^{d}$ concentrates near the norm one vectors. More precisely, let $X$ be a random vector, chosen uniformly from $[-s, 1]^{d}$. Then its coordinate functions $X_{i}$ are independent random variables, uniformly distributed over $[-s, 1]$. By slight abuse of notation 
we use $P$ to denote both uniform probabilities on $[-s, 1]^{d}$ and on $[-s, 1]$. Fix $0<\varepsilon<1$, set $t=1$, and choose $\delta \in(0, \varepsilon)$ so that $\omega(1-\delta) / \omega(1)>1-\varepsilon$. Then, for any $0 \leq s \leq 1$ and all sufficiently high $d$, we have

$$
\begin{gathered}
P\left(\|X\|_{\infty}>1-\delta\right) \geq P\left(\max \left\{X_{1}, \ldots, X_{d}\right\}>1-\delta\right)=1-\Pi_{1}^{d} P\left(X_{i} \leq 1-\delta\right) \\
=1-\left(\frac{1+s-\delta}{1+s}\right)^{d} \geq 1-\left(1-\frac{\delta}{2}\right)^{d}>1-\varepsilon .
\end{gathered}
$$

Thus, if the dimension $d$ is large enough (depending of $\omega$ and $\varepsilon$ ) it follows that

$$
1 \geq\|M\|_{\mathrm{Op}(\omega)} \geq \inf _{0 \leq s \leq 1} \frac{1}{\omega(1)} \int_{[-s, 1]^{d}} \omega\left(\|x\|_{\infty}\right) \frac{d x}{(1+s)^{d}} \geq(1-\varepsilon) \frac{\omega(1-\delta)}{\omega(1)}>(1-\varepsilon)^{2} .
$$

Observe that in the above proof we did not need to establish how the optimal value $s_{d}$ of $s \in[0,1]$ behaves as $d \rightarrow \infty$. Intuition suggests that since the measure of $\left[-s_{d}, 1\right]^{d}$ concentrates near its border as $d$ grows, in order to minimize the average value of the norm over this cube, the origin should be increasingly closer to the boundary of $\left[-s_{d}, 1\right]^{d}$. Or, in other words, $s_{d}$ should approach 0 as $d \rightarrow \infty$. This intuition is, in fact, completely erroneous, as formula (3.2.2) below shows (see also the proof of Corollary 3.4): When $d \rightarrow \infty$, the optimal $s_{d}$ tends to 1 , and thus the optimal averaging cube has $\ell_{\infty}$ diameter approaching 2. Nevertheless, we shall show that in the euclidean case the above intuition is correct: As $d \rightarrow \infty$ the origin must indeed be increasingly closer to the boundary of the optimal ball, in order to minimize the average value of the norm, and the $\ell_{2}$ diameter of the optimal averaging ball must approach 1 rather than 2 . This helps to understand why the asymptotic behavior of $\|M\|_{\mathrm{Op}(1)}$ is so different in the $\ell_{\infty}$ and $\ell_{2}$ cases.

Next we specialize the preceding theorem to the Lipschitz and Hölder functions, obtaining the following corollary.

Corollary 3.2. Let $\alpha \in(0,1]$, and consider the space $\left(\mathbb{R}^{d},\|\cdot\|_{\infty}\right)$. Then, on $\operatorname{Lip}_{\alpha}\left(\mathbb{R}^{d},\|\cdot\|_{\infty}\right)$,

$$
\|M\|_{\mathrm{Op}(\alpha)}=\min _{0 \leq s \leq 1}\left\{\frac{d}{(1+s)^{d}}\left[\frac{2^{d} s^{\alpha+d}}{\alpha+d}+\sum_{j=0}^{d-1}\left(\begin{array}{c}
d-1 \\
j
\end{array}\right) \frac{s^{d-1-j}-s^{d+\alpha}}{\alpha+j+1}\right]\right\} .
$$

In particular, when $\alpha=1$, that is, for Lipschitz functions on $\left(\mathbb{R}^{d},\|\cdot\|_{\infty}\right)$ we have

$$
\|M\|_{\mathrm{Op}(1)}=\frac{d}{d+1}-\frac{1}{d+1} \max _{0<s<1}\left\{s-\frac{2^{d} s^{d+1}}{(1+s)^{d}}\right\} .
$$

Proof. To obtain (3.2.1), use (3.1.2) in Theorem 3.1 with $\omega(t)=t^{\alpha}$, and integrate. As for (3.2.2), it does not seem to be easy to derive it from (3.2.1) by substituting $\alpha=1$. Instead, use (3.1.2) again, evaluating the integral $\int_{s}^{1}(u+s)^{d-1} u d u$ via the change of variable $v=u+s$. This yields (3.2.2) but with $\max _{0 \leq s \leq 1}$. To further refine this expression and obtain $\max _{0<s<1}$, we observe that the maximum is actually achieved at some interior point of the unit interval. This can be seen by writing $g_{d}(s):=s-2^{d} s^{d+1} /(1+s)^{d}$ for $s \in[0,1]$, and noting that since 
$g_{d}(0)=g_{d}(1)=0$, and $g_{d}^{\prime \prime}<0$ on $(0,1)$, the function is strictly concave there. Thus, it has a unique maximum, which must occur at some interior point.

Remark 3.3. Specializing formula (3.2.1) to $d=1$ and $d=2$ gives the following expressions. We mention that they can also be obtained easily and directly from (3.1.1).

When $d=1$, we find that for every $f \in \operatorname{Lip}_{\alpha}(\mathbb{R})$ and every $\alpha \in(0,1]$,

$$
\|M\|_{\mathrm{Op}(\alpha)}=\min _{0<s<1}\left\{\frac{1+s^{1+\alpha}}{(1+\alpha)(1+s)}\right\} .
$$

Furthermore, this result is independent of the $\mathbb{R}$ norm, since $d=1$. The fact that the unique minimum occurs in the interior of $(0,1)$ is shown, as above, by elementary calculus arguments: Fix $\alpha \in(0,1]$, and for $s \in[0,1]$ write $g_{\alpha}(s):=\frac{1+s^{\alpha+1}}{(\alpha+1)(1+s)}$. Evaluating $g_{\alpha}$ on 0 and 1 we see (by inspection) that it achieves its maximum value at these points. Since $g_{\alpha}^{\prime \prime}>0$ on $(0,1)$, the function is strictly convex there, and thus it has a unique minimum.

It is clear from (3.3.1) that on the real line, $\|M\|_{\mathrm{Op}(\alpha)}<(1+\alpha)^{-1}$, which is the sharp bound in the local case, that is, for Hölder functions on intervals (cf. Corollary 4.5 below). Thus, Hölder constants are smaller on the line than on proper subintervals. Note also that as $\alpha \rightarrow 0,\|M\|_{\mathrm{Op}(\alpha)} \rightarrow 1$, again by (3.3.1). Convergence to 1 as $\alpha \rightarrow 0$ holds also in dimension 2 , by (3.3.2) below.

When $d=2$, formulas (3.2.1) and (3.1.1) become

$$
\|M\|_{\mathrm{Op}(\alpha)}=\min _{0 \leq s \leq 1} 2\left(\frac{\alpha+1+(\alpha+2) s+(2 \alpha+1) s^{\alpha+2}}{(\alpha+1)(\alpha+2)(1+s)^{2}}\right) .
$$

Here calculus arguments regarding extrema are more involved, and in fact, as $d$ grows the formulas given by (3.2.1) become less manageable, though of course, numerical estimation is possible. However, in the simpler Lipschitz case, where $\alpha=1$, there is still a good deal of explicit information that can be extracted from (3.2.2). Contrary to our usual notation, in the next corollary we shall indicate the dependency of the maximal operator on the dimension $d$ by writing $M_{d}$.

Corollary 3.4. On $\operatorname{Lip}\left(\mathbb{R}^{d},\|\cdot\|_{\infty}\right),\left\|M_{d}\right\|_{\mathrm{Op}(1)}=(d-1) /(d+1)+o(1 /(d+1))$. More precisely,

$$
\frac{d-1}{d+1}<\left\|M_{d}\right\|_{\mathrm{Op}(1)} \leq \frac{d}{d+1}-\frac{1}{d+1}\left(1-\frac{1}{\sqrt{d}}\right)\left[1-\left(1-\frac{1}{2 \sqrt{d}-1}\right)^{d}\right] \text {. }
$$

Proof. The lower bound follows from (3.2.2) by noticing that $g_{d}(s):=s-2^{d} s^{d+1} /(1+s)^{d}<$ $s \leq 1$ for all $s \in(0,1]$. To get an upper bound it is enough to take (3.2.2) and assign any value from $[0,1]$ to $s$. For instance, the choices $s=0$ and $s=1$ recover the general bound $d /(d+1)$ from Corollary 2.13 (so $\left.\left\|M_{d}\right\|_{\mathrm{Op}(1)}=(d-1) /(d+1)+O(1 /(d+1))\right)$, but of course one can do better: The right hand side of (3.4.1) is obtained by taking $s=1-1 / \sqrt{d}$.

While sufficient to prove asymptotic equivalence, the choice $s=1-1 / \sqrt{d}$ made above is somewhat arbitrary and can easily be improved, at the cost of getting more complicated 
upper bounds (so the second inequality in (3.4.1) is also strict). The optimal choice is the unique solution to the polynomial equation given next.

Lemma 3.5. The norm of the maximal operator $M$ on $\operatorname{Lip}\left(\mathbb{R}^{d},\|\cdot\|_{\infty}\right)$ is obtained by evaluating

$$
h_{d}(s):=\frac{d}{d+1}-\frac{1}{d+1}\left(s-\frac{2^{d} s^{d+1}}{(1+s)^{d}}\right)
$$

on the unique solution $s_{d}$ inside $(0,1)$ of the polynomial equation

$$
p_{d}(s):=2^{d} s^{d+1}-(1+s)^{d+1}+2^{d}(d+1) s^{d}=0 .
$$

Proof. Formula (3.5.1) follows immediately from (3.2.2). Recall from the proof of Corollary 3.2 that the function $g_{d}(s):=s-2^{d} s^{d+1} /(1+s)^{d}$ has a unique maximum on $(0,1)$, to be found by solving $g_{d}^{\prime}=0$, or equivalently, $p_{d}(s):=(1+s)^{d+1}-2^{d}(d+1) s^{d}-2^{d} s^{d+1}=0$, on the said interval.

When $d \leq 3, \operatorname{deg} p_{d} \leq 4$, so its roots can be found explicitly using Cardan's formula. We do this next, thereby obtaining the sharp constant in dimension 1 , and for the $\ell_{\infty}$ norm, the sharp constants in dimensions 2 and 3. Details are included for the readers convenience.

Corollary 3.6. On $\operatorname{Lip}(\mathbb{R})$ we have $\|M\|_{\mathrm{Op}(1)}=\sqrt{2}-1$.

Proof. Solving $p_{1}(s)=1-2 s-2 s^{2}=0$ on $(0,1)$, we find that $h_{1}\left(s_{1}\right)=h_{1}(\sqrt{2}-1)=\sqrt{2}-1$.

Corollary 3.7. On $\operatorname{Lip}\left(\mathbb{R}^{2},\|\cdot\|_{\infty}\right)$ we have

$$
\|M\|_{\mathrm{Op}(1)}=\frac{4}{\sqrt{3}} \cos \left(\frac{5 \pi}{18}\right)+\sqrt{3} \sec \left(\frac{5 \pi}{18}\right)-\frac{1}{4} \sec ^{2}\left(\frac{5 \pi}{18}\right)-3 .
$$

Proof. We use Lemma 3.5, finding first the unique root of $s^{3}+3 s^{2}-s-1 / 3$ in $(0,1)$. The change of variable $s \mapsto s-1$ leads to the reduced form $s^{3}-4 s+8 / 3$. Since $(8 / 3)^{2}-4^{4} / 3^{3}=-64 / 27<0$, this is the irreducible case in Cardan's formula. Following Viète we write $s=(4 / \sqrt{3}) y$, to obtain $4 y^{3}-3 y=-\sqrt{3} / 2=\cos (5 \pi / 6+2 \pi k)$, and now we use the trigonometrical identity $4 \cos ^{3}(\vartheta)-3 \cos (\vartheta)=\cos (3 \vartheta)$ to conclude that $s=(4 / \sqrt{3}) \cos (5 \pi / 18+2 k \pi / 3), k=0,1,2$. Of these three roots only the one corresponding to $k=0$ belongs to $(1,2)$, so $s_{2}=\frac{4}{\sqrt{3}} \cos \frac{5 \pi}{18}-$ $1 \in(0,1)$. Finally, evaluating $h_{2}(\mathrm{cf}$. (3.5.1) $)$ on $s_{2}$ and simplifying once more we find that

$$
h_{2}\left(s_{2}\right)=\frac{4}{\sqrt{3}} \cos \left(\frac{5 \pi}{18}\right)+\sqrt{3} \sec \left(\frac{5 \pi}{18}\right)-\frac{1}{4} \sec ^{2}\left(\frac{5 \pi}{18}\right)-3 .
$$

Remark 3.8. Therefore $\|M\|_{\mathrm{Op}(1)} \approx 0.574$ on $\operatorname{Lip}\left(\mathbb{R}^{2},\|\cdot\|_{\infty}\right)$.

Remark 3.9. In dimension two, the results (3.3.2) and (3.7.1) hold verbatim if we use the $\ell_{1}$ norm instead of the $\ell_{\infty}$ norm, since in dimension two $\ell_{1}$ balls are just rotated cubes. In fact, this phenomenon repeats itself in every dimension, despite the fact that the geometry is very different when $d \geq 3$. Thus, the next result holds also for the $\ell_{1}$ norm. 
Corollary 3.10. On $\operatorname{Lip}\left(\mathbb{R}^{3},\|\cdot\|_{\infty}\right)$ we have

$$
\|M\|_{\mathrm{Op}(1)}=1-\frac{\frac{2^{9 / 4}}{\left((\sqrt{8}+\sqrt{7})^{1 / 3}+(\sqrt{8}-\sqrt{7})^{1 / 3}\right)^{3 / 2}}}{\left(1+\left(\frac{2^{9 / 4}}{\left((\sqrt{8}+\sqrt{7})^{1 / 3}+(\sqrt{8}-\sqrt{7})^{1 / 3}\right)^{3 / 2}}-1\right)^{1 / 2}\right)^{3}} .
$$

Proof. By Lemma 3.5, it is enough to find the the unique root $s_{3}$ of $p_{3}(s)=7 s^{4}+28 s^{3}-$ $6 s^{2}-4 s-1=0$ in $(0,1)$ and then evaluate $h_{3}\left(s_{3}\right)$ (see (3.5.1)). Using the change of variable $s=1 /(2 t-1)$, we note that $0<s<1$ if and only if $t>1$, and $p_{3}(s)=0$ if and only if $2 t^{4}-8 t+3=0$. Now it can be checked by direct substitution that

$$
t_{3}=\frac{\left((\sqrt{8}+\sqrt{7})^{1 / 3}+(\sqrt{8}-\sqrt{7})^{1 / 3}\right)^{1 / 2}}{2^{3 / 4}}\left(1+\left(\frac{2^{9 / 4}}{\left((\sqrt{8}+\sqrt{7})^{1 / 3}+(\sqrt{8}-\sqrt{7})^{1 / 3}\right)^{3 / 2}}-1\right)^{1 / 2}\right)
$$

satisfies $2 t^{4}-8 t+3=0$, and furthermore, it is the unique $t>1$ with this property, by the uniqueness of $s_{3}$ in $(0,1)$. Rather than substituting the value of $s_{3}$ directly in $h_{3}$, it is more convenient to simplify $h_{3}\left(s_{3}\right)$ first. Note that

$$
h_{3}\left(s_{3}\right)=\frac{3+8 s_{3}+6 s_{3}^{2}+7 s_{3}^{4}}{4\left(1+s_{3}\right)^{3}},
$$

and also $7 s_{3}^{4}=-28 s_{3}^{3}+6 s_{3}^{2}+4 s_{3}+1$, since $p_{3}\left(s_{3}\right)=0$. Eliminating the fourth order term and simplifying we get

$$
\|M\|_{\mathrm{Op}(1)}=h_{3}\left(s_{3}\right)=1-8\left(\frac{s_{3}}{1+s_{3}}\right)^{3} .
$$

Using $s_{3}=1 /\left(2 t_{3}-1\right)$, the preceding equality becomes $\|M\|_{\mathrm{Op}(1)}=1-t_{3}^{-3}$, and now (3.10.1) follows by substituting in the numerical value of $t_{3}$.

Remark 3.11. $\|M\|_{\mathrm{Op}(1)} \approx 0.66155$ on $\operatorname{Lip}\left(\mathbb{R}^{3},\|\cdot\|_{\infty}\right)$.

Next we study the cases $p=2$ and $p=1$. Since in one dimension all the $\ell_{p}$ unit balls coincide with the interval $[-1,1]$, the case $d=1$ is covered by Theorem 3.1 .

Lemma 3.12. Let $d \geq 2$, let $f:[0, \infty) \rightarrow[0, \infty)$ be an increasing function, and let $1 \leq p<$ $\infty$. Then, for all $R>0$, all $c=\left(c_{1}, \ldots, c_{d}\right) \in \mathbb{R}^{d}$, and all $a \in \mathbb{R}$ such that $|a| \leq\left|c_{1}\right|$, we have

$$
\int_{B\left(a e_{1}, R\right)} f\left(\|x\|_{p}^{p}\right) d x \leq \int_{B(c, R)} f\left(\|x\|_{p}^{p}\right) d x .
$$

Proof. Write $\hat{c}=\left(c_{2}, \ldots, c_{d}\right)$ and $\hat{x}=\left(x_{2}, \ldots, x_{d}\right)$. Using Fubini's Theorem and Anderson's Theorem (cf. Remark 2.8) in $d$ and $d-1$ dimensions, we get

$$
\begin{gathered}
\int_{B(c, R)} f\left(\|x\|_{p}^{p}\right) d x=\int_{c_{1}-R}^{c_{1}+R}\left(\int_{B\left(\hat{c},\left(R^{p}-\left|c_{1}-x_{1}\right|^{p}\right)^{1 / p}\right)} f\left(\left|x_{1}\right|^{p}+\|\hat{x}\|_{p}^{p}\right) d \hat{x}\right) d x_{1} \geq \\
\int_{c_{1}-R}^{c_{1}+R}\left(\int_{B\left(0,\left(R^{p}-\left|c_{1}-x_{1}\right|^{p}\right)^{1 / p}\right)} f\left(\left|x_{1}\right|^{p}+\|\hat{x}\|_{p}^{p}\right) d \hat{x}\right) d x_{1}=\int_{B\left(c_{1} e_{1}, R\right)} f\left(\|x\|_{p}^{p}\right) d x \geq
\end{gathered}
$$




$$
\int_{B\left(a e_{1}, R\right)} f\left(\|x\|_{p}^{p}\right) d x
$$

To apply (2.14.1), it is useful to determine on which unit vectors the supremum is attained. We have seen that one such vector in the $\ell_{\infty}$ case is $(1, \ldots, 1)$. For other $p$ norms, if $e_{1}$ can be selected, this usually leads to simplification of the formulas. The next lemma reduces the question of the optimality of $e_{1}$ to the two dimensional case.

Lemma 3.13. Let $f:[0, \infty) \rightarrow[0, \infty)$ be an increasing function, and let $1 \leq p<\infty$. If for all $c \in \mathbb{R}^{2}$ and all $R \in(0,1]$ we have

$$
\int_{B(c, R)} f\left(\|x\|_{p}^{p}\right) d x \leq \int_{B\left(\|c\|_{p} e_{1}, R\right)} f\left(\|x\|_{p}^{p}\right) d x
$$

then for all $d \geq 2$, all $c \in \mathbb{R}^{d}$, and all $R \in(0,1]$,

$$
\int_{B(c, R)} f\left(\|x\|_{p}^{p}\right) d x \leq \int_{B\left(\|c\|_{p} e_{1}, R\right)} f\left(\|x\|_{p}^{p}\right) d x .
$$

Proof. Assume the result is true for $d \geq 2$. Let $\bar{c} \in \mathbb{R}^{d+1}$ and let $R>0$. We write $\bar{c}=(c, b)$, where $c \in \mathbb{R}^{d}, \bar{x}=(x, y), x \in \mathbb{R}^{d}$, and $x=\left(x_{1}, \hat{x}\right), \hat{x}=\left(x_{2}, \ldots, x_{d}\right) \in \mathbb{R}^{d-1}$. From Fubini's Theorem, induction, and the assumption for $d=2$, we get

$$
\begin{gathered}
\int_{B(\bar{c}, R)} f\left(\|\bar{x}\|_{p}^{p}\right) d \bar{x}=\int_{b-R}^{b+R} \int_{\left|x_{1}-c_{1}\right|^{p}+\ldots+\left|x_{d}-c_{d}\right|^{p} \leq R^{p}-|y-b|^{p}} f\left(\|x\|_{p}^{p}+|y|^{p}\right) d x d y \\
\leq \int_{b-R}^{b+R} \int_{\left|x_{1}-\|c\|_{p}\right|^{p}+\left|x_{2}\right|^{p}+\ldots+\left|x_{d}\right|^{p} \leq R^{p}-|y-b|^{p}} f\left(\|x\|_{p}^{p}+|y|^{p}\right) d x d y \\
=\int_{\|\hat{x}\|_{p}^{p} \leq R^{p}}\left(\int_{\left|x_{1}-\|c\|_{p}\right|^{p}+|y-b|^{p} \leq R^{p}-\|\hat{x}\|_{p}^{p}} f\left(\|\hat{x}\|_{p}^{p}+\left|x_{1}\right|^{p}+|y|^{p}\right) d x_{1} d y\right) d \hat{x} \\
\leq \int_{\|\hat{x}\|_{p}^{p} \leq R^{p}}\left(\int_{\left|x_{1}-\|\bar{c}\|_{p}\right|^{p}+|y|^{p} \leq R^{p}-\|\hat{x}\|_{p}^{p}} f\left(\|\hat{x}\|_{p}^{p}+\left|x_{1}\right|^{p}+|y|^{p}\right) d x_{1} d y\right) d \hat{x} \\
=\int_{B\left(\|\bar{c}\|_{p} e_{1}, R\right)} f\left(\|\bar{x}\|_{p}^{p}\right) d \bar{x} .
\end{gathered}
$$

For euclidean balls, optimality of $e_{1}$, or any other vector (in every dimension), follows from symmetry. Now let $d=2$. For $\ell_{1}$ balls, optimality of $e_{1}$ follows from the optimality of $(1,1)$ for $\ell_{\infty}$ balls, since each unit ball and its corresponding norm can be obtained from the other via a rotation and a dilation. And in this case $e_{1}$ is strictly better than nearby vectors. We have not been able to prove in a direct way the optimality of $e_{1}$ for other $\ell_{p}$ balls, $1<p<2$, even for explicit moduli (approximation arguments seem to yield very limited results). 
It is well known, and we use it below in the cases $p=1,2$, that when $1 \leq p<\infty$ the measure of the unit ball concentrates near the vectors with norm one and first coordinate equal to zero, that is, near the "vertical equator" perpendicular to $e_{1}:\left\{x \in \mathbb{R}^{d}: x_{1}=0\right.$ and $\left.\|x\|_{p}=1\right\}$. Indeed, the sections of the unit ball perpendicular to $e_{1}$ are balls in $\mathbb{R}^{d-1}$, and a small decrease in the radius $r$ causes a large decrease in mass whenever $d$ is high, since Lebesgue measure in $\mathbb{R}^{d-1}$ scales like $r^{d-1}$. It follows that the measure of the unit ball concentrates on the sections of maximal radius, i.e., when $x_{1} \approx 0$. Likewise, the measure of the unit ball concentrates near the unit sphere. Thus we have concentration near the vertical equator, since the intersection of two very large subsets of the unit ball must be large. Of course, when $p=\infty$ this concentration near the vertical equator does not takes place.

Let $\mathbb{B}_{p}^{d}$ be the $\ell_{p}$ unit ball $\left\{x \in \mathbb{R}^{d}:\|x\|_{p} \leq 1\right\}$ (for convenience here we take balls to be closed), let $\mathbb{S}_{p}^{d-1}$ be the corresponding unit sphere, given by $\left\{x \in \mathbb{R}^{d}:\|x\|_{p}=1\right\}$, and let $\left|\mathbb{B}_{p}^{d}\right|$ and $\left|\mathbb{S}_{p}^{d-1}\right|$ be their respective $d$ and $d-1$ volumes. When considering radii $r$ not necessarily equal to one, we write $\mathbb{B}_{p}^{d}(r)$ and $\mathbb{S}_{p}^{d-1}(r)$.

Theorem 3.14. Let $d \geq 2$, let $M$ be the uncentered maximal operator associated to balls defined by the $\ell_{2}$ norm, i.e., to euclidean balls, and let $\omega$ be a modulus of continuity. Then

$$
\begin{gathered}
\|M\|_{\mathrm{Op}(\omega)}=\sup _{t>0} \inf _{1 / 2 \leq R \leq 1} \frac{(d-1) \Gamma(1+d / 2)}{\omega(t) \sqrt{\pi} \Gamma(1 / 2+d / 2)} \times \\
\int_{-1}^{1} \int_{0}^{\left(1-u_{1}^{2}\right)^{1 / 2}} \omega\left(t\left(\left(1-R+R u_{1}\right)^{2}+R^{2} \rho^{2}\right)^{1 / 2}\right) \rho^{d-2} d \rho d u_{1} .
\end{gathered}
$$

Furthermore, if we select the same modulus $\omega$ in all dimensions, we have

$$
\sup _{t>0}\left\{\frac{\omega\left(2^{-\frac{1}{2}} t\right)}{\omega(t)}\right\} \leq \liminf _{d \rightarrow \infty}\|M\|_{\mathrm{Op}(\omega)}
$$

and

$$
\limsup _{d \rightarrow \infty}\|M\|_{\mathrm{Op}(\omega)} \leq \inf _{r>1} \sup _{t>0}\left\{\frac{\omega\left(2^{-\frac{1}{2}} r t\right)}{\omega(t)}\right\} .
$$

Under the additional assumption that $\omega$ is concave, the limit exists, it is equal to the left hand side of (3.14.2), and bounds $\|M\|_{\mathrm{Op}(\omega)}$ uniformly in $d$ : Given $d \geq 1$,

$$
\|M\|_{\mathrm{Op}(\omega)} \leq \sup _{t>0}\left\{\frac{\omega\left(2^{-\frac{1}{2}} t\right)}{\omega(t)}\right\}=\lim _{d \rightarrow \infty}\|M\|_{\mathrm{Op}(\omega)} .
$$

In particular, for the Hölder and Lipschitz classes we obtain

$$
\|M\|_{\mathrm{Op}(\alpha)} \leq 2^{-\frac{\alpha}{2}} \quad \text { for all } d \geq 1, \text { and } \quad \lim _{d \rightarrow \infty}\|M\|_{\mathrm{Op}(\alpha)}=2^{-\frac{\alpha}{2}} .
$$


In order to give an idea about the size of the constant term (for $d$ fixed) in (3.14.1), we point out that

$$
\left(\frac{d}{2}\right)^{1 / 2} \leq \frac{\Gamma(1+d / 2)}{\Gamma(1 / 2+d / 2)} \leq\left(\frac{d+1}{2}\right)^{1 / 2}
$$

This is an easy consequence of the log-convexity of the $\Gamma$ function (cf. Exercise 5, pg. 216 of [Web] $)$. Note also that if instead of taking the infimum in the right hand side of (3.14.1) we just set $R=1$, we are averaging over the whole unit ball, that is, we are acting as in the proof of Corollary 2.13. And indeed, if we change to polar coordinates and integrate we recover the bound $d /(d+1)$. So here, a better choice of $R$ leads to lower asymptotic bounds. We shall see that in fact, when $d \rightarrow \infty$ the optimal $R=R(d)$ approaches $1 / 2$, as intuition suggests. The $s$-dimensional Hausdorff measure on $\mathbb{R}^{d}$ is denoted by $\mathcal{H}^{s}$.

Proof. As in the case of Theorem 3.1 , it is enough to consider balls contained in $\mathbb{B}_{2}^{d}$. To see this, fix any unit vector $v$, and let $B(c, R)$ be a minimizing ball for $v$ in (2.14). Suppose $B(c, R)$ has points outside $\mathbb{B}_{2}^{d}$. Translating $B(c, R)$ towards the origin along the ray $\{t c: t \geq 0\}$ determined by the vector $c$, so that the displaced ball $B\left(c^{\prime}, R\right)$ is fully contained in $\mathbb{B}_{2}^{d}$ and tangent to the unit sphere, leads to

$$
\int_{B(0,1)} \omega\left(t\left\|c^{\prime}+R u\right\|_{2}\right) d u \leq \int_{B(0,1)} \omega\left(t\|c+R u\|_{2}\right) d u
$$

by Anderson's Theorem (see Remark 2.8). It may well happen that after the translation $v \notin B\left(c^{\prime}, R\right)$. If so, rotate $B\left(c^{\prime}, R\right)$ about the origin to make its new center lie in the segment $[0, v]$. Since this does not change the value of the integral, we conclude that it suffices to consider balls contained in $\mathbb{B}_{2}^{d}$. Again by rotational symmetry we may take $v$ to be $e_{1}$, so it is enough to consider centers $c=(1-R) e_{1}$ and radii $R$, with $0 \leq R \leq 1$. Hence, by (2.14.1) we have

$$
\|M\|_{\mathrm{Op}(\omega)}=\sup _{t>0} \inf _{0 \leq R \leq 1} \frac{1}{\omega(t)} \int_{\mathbb{B}_{2}^{d}} \omega\left(t\left\|(1-R) e_{1}+R u\right\|_{2}\right) \frac{d u}{\left|\mathbb{B}_{2}^{d}\right|} .
$$

Note that the infimum in (3.14.6) is attained when $R \in[1 / 2,1]$. In fact, we claim that the function $f_{2}(R):=\left\|(1-R) e_{1}+R u\right\|_{2}^{2}$ is decreasing when $R \leq 1 / 2$, so the minimum must indeed occur on $1 / 2 \leq R \leq 1$. Differentiating $f_{2}(R)=\left(1-R+R u_{1}\right)^{2}+R^{2} \sum_{i=1}^{d} u_{i}^{2}-R^{2} u_{1}^{2}$, and using $\sum_{i=1}^{d} u_{i}^{2} \leq 1$ together with $R \leq 1 / 2$, the claim follows.

Given $u \in \mathbb{B}_{2}^{d}$, we write $u=\left(u_{1}, y\right)$, where $u_{1} \in \mathbb{R}$ and $y \in \mathbb{R}^{d-1}$. Denote by $P_{d}$ the uniform probability on $\mathbb{B}_{2}^{d}$, so $d P_{d}(u)=d u /\left|\mathbb{B}_{2}^{d}\right|$. To prove (3.14.3) and (3.14.2), fix $t>0$, and note that by concentration of measure near the vertical equator, for each $\delta>0$ we have

$$
\lim _{d \rightarrow \infty} P_{d}\left(\left\{\left|u_{1}\right|<\delta, 1-\delta<\|y\|_{2} \leq 1\right\}\right)=1
$$

Of course, the weaker assertion

$$
\lim _{d \rightarrow \infty} P_{d}\left(\left\{\left|u_{1}\right|<\delta\right\}\right)=1
$$


also holds. Since $\left\|(1-R) e_{1}+R u\right\|_{2} \leq 1$ for all $u \in \mathbb{B}_{2}^{d}$ and all $R \in[1 / 2,1]$,

$$
\inf _{1 / 2 \leq R \leq 1} \int_{\mathbb{B}_{2}^{d} \backslash\left\{\left|u_{1}\right|<\delta\right\}} \frac{\omega\left(t\left\|(1-R) e_{1}+R u\right\|_{2}\right)}{\omega(t)} d P_{d}(u) \leq P_{d}\left(\left\{\left|u_{1}\right| \geq \delta\right\}\right) .
$$

Next, note that on $\mathbb{B}_{2}^{d} \cap\left\{\left|u_{1}\right|<\delta\right\}$, for every $d$ and every $R \in[1 / 2,1]$ we have

$$
\left\|(1-R) e_{1}+R u\right\|_{2} \leq\left((1-R+\delta)^{2}+R^{2}\right)^{1 / 2} .
$$

The unique minimum of $h_{1}(R):=(1-R+\delta)^{2}+R^{2}$ on $[1 / 2,1]$ is attained at $R_{\delta}=(1+\delta) / 2$, and there $h_{1}\left(R_{\delta}\right)=(1+\delta)^{2} / 2$. Thus,

$$
\left\|\left(1-R_{\delta}\right) e_{1}+R_{\delta} u\right\|_{2} \leq 2^{-1 / 2}(1+\delta),
$$

so splitting $\mathbb{B}_{2}^{d}$ into the regions where $\left|u_{1}\right|<\delta$ and $\left|u_{1}\right| \geq \delta$ we obtain

$$
\inf _{1 / 2 \leq R \leq 1} \int_{\mathbb{B}_{2}^{d}} \frac{\omega\left(t\left\|(1-R) e_{1}+R u\right\|_{2}\right)}{\omega(t)} d P_{2}(u) \leq \frac{\omega\left(t\left(2^{-1 / 2}(1+\delta)\right)\right)}{\omega(t)}+P_{d}\left(\left\{\left|u_{1}\right| \geq \delta\right\}\right) .
$$

Taking on both sides of the preceding inequality first $\sup _{t>0}$, second, $\lim \sup _{d \rightarrow \infty}$, and third, $\inf _{\delta>0}$, from (3.14.6) (with $R \in[1 / 2,1]$ ) we get

$$
\limsup _{d \rightarrow \infty}\|M\|_{\mathrm{Op}(\omega)} \leq \inf _{\delta>0} \sup _{t>0} \frac{\omega\left(2^{-\frac{1}{2}}(1+\delta) t\right)}{\omega(t)} .
$$

This proves (3.14.3).

The argument used to obtain (3.14.2) is similar. Fix $u=\left(u_{1}, y\right) \in \mathbb{R}^{d}$ and note that on $\mathbb{R}$ the function

$$
h_{2}(R):=\left(1-R+R u_{1}\right)^{2}+R^{2}\|y\|_{2}^{2}=\left\|(1-R) e_{1}+R u\right\|_{2}^{2}
$$

achieves its unique minimum at $R_{u}=\left(1-u_{1}\right) /\left[\left(1-u_{1}\right)^{2}+\|y\|_{2}^{2}\right]$, where it takes the value $h_{2}\left(R_{u}\right)=\|y\|_{2}^{2} /\left[\left(1-u_{1}\right)^{2}+\|y\|_{2}^{2}\right]$. Thus, for $0<\delta<1$ and $u \in \mathbb{B}_{2}^{d} \cap\left\{\left|u_{1}\right|<\delta, 1-\delta<\|y\|_{2} \leq 1\right\}$ we have

$$
\frac{1-\delta}{\sqrt{(1+\delta)^{2}+1}} \leq \frac{\|y\|_{2}}{\sqrt{\left(1-u_{1}\right)^{2}+\|y\|_{2}^{2}}} \leq\left\|(1-R) e_{1}+R u\right\|_{2},
$$

from which it follows that

$$
\begin{aligned}
& \sup _{t>0} P_{d}\left(\left\{\left|u_{1}\right|<\delta, 1-\delta<\|y\|_{2} \leq 1\right\}\right) \frac{\omega\left(t\left((1+\delta)^{2}+1\right)^{-1 / 2}(1-\delta)\right)}{\omega(t)} \\
& \leq \sup _{t>0} \inf _{1 / 2 \leq R \leq 1} \int_{\mathbb{B}_{2}^{d}} \frac{\omega\left(t\left\|(1-R) e_{1}+R u\right\|_{2}\right)}{\omega(t)} d P_{d}(u)=\|M\|_{\mathrm{Op}(\omega)} .
\end{aligned}
$$


Now (3.14.14) increases with $d$, so taking the limit inferior in (3.14.14) and (3.14.15) as $d \rightarrow \infty$, we get

$$
\sup _{t>0} \frac{\omega\left(t\left((1+\delta)^{2}+1\right)^{-1 / 2}(1-\delta)\right)}{\omega(t)} \leq \liminf _{d \rightarrow \infty}\|M\|_{\mathrm{Op}(\omega)} .
$$

Likewise, the left hand side of the preceding inequality is decreasing in $\delta \in(0,1)$, so (3.14.2) follows by taking the supremum over $\delta$, interchanging it with the supremum over $t$, and letting $\delta \downarrow 0$.

Next, write $u=\left(u_{1}, y\right) \in \mathbb{R}^{d}$ and $y=\rho \eta$, where $\rho=\|y\|_{2}$ and $\|\eta\|_{2}=1$. By Fubini's Theorem

$$
\int_{\mathbb{B}_{2}^{d}} \omega\left(t\left\|(1-R) e_{1}+R u\right\|_{2}\right) d u=\int_{-1}^{1} \int_{\mathbb{B}_{2}^{d-1}\left(\left(1-\left|u_{1}\right|^{2}\right)^{1 / 2}\right)} \omega\left(t\left\|(1-R) e_{1}+R\left(u_{1}, y\right)\right\|_{2}\right) d y d u_{1} .
$$

Using polar coordinates on the vertical sections, or equivalently, by the coarea formula, we get

$$
\begin{gathered}
\int_{\mathbb{B}_{2}^{d-1}\left(\left(1-u_{1}^{2}\right)^{1 / 2}\right)} \omega\left(t\left\|(1-R) e_{1}+R\left(u_{1}, y\right)\right\|_{2}\right) d y \\
=\int_{0}^{\left(1-u_{1}^{2}\right)^{1 / 2}} \int_{\mathbb{S}_{2}^{d-2}(\rho)} \omega\left(\left(\left|(1-R)+R u_{1}\right|^{2}+R^{2}\|y\|_{2}^{2}\right)^{1 / 2}\right) d \mathcal{H}^{d-2}(y) d \rho \\
=(d-1)\left|\mathbb{B}_{2}^{d-1}\right| \int_{0}^{\left(1-u_{1}^{2}\right)^{1 / 2}} \omega\left(\left(\left|(1-R)+R u_{1}\right|^{2}+R^{2} \rho^{2}\right)^{1 / 2}\right) \rho^{d-2} d \rho .
\end{gathered}
$$

Since $\left|\mathbb{B}_{2}^{d}\right|=\frac{\pi^{d / 2}}{\Gamma(1+d / 2)}$, (3.14.1) follows from $(\underline{3.14 .6})$ and the preceding equalities.

Suppose next that $\omega$ is concave. We show that the inequality in (3.14.4) holds for every $d \geq 1$. When $d=1$ the result follows from (3.1.1) and the concavity of $\omega$ :

$$
\|M\|_{\mathrm{Op}(\omega)} \leq \sup _{t>0} \frac{1}{\omega(t)} \int_{0}^{1} \omega(t x) d x \leq \sup _{t>0} \frac{1}{\omega(t)} \omega\left(t \int_{0}^{1} x d x\right)=\sup _{t>0} \frac{\omega(t / 2)}{\omega(t)} .
$$

Let $d>1$ and let $t>0$ be fixed. If $\omega(z)$ is concave, then so is $\omega\left(t z^{1 / 2}\right)$. Thus, given $x \geq y \geq 0$, we have $\omega\left((x-y)^{1 / 2}\right)+\omega\left((x+y)^{1 / 2}\right) \leq 2 \omega\left(x^{1 / 2}\right)$. We write the integral appearing in (3.14.1), together with its constant term, as

$$
I=\int_{-1}^{1} \int_{0}^{\left(1-u_{1}^{2}\right)^{1 / 2}} \omega\left(t\left((1-R)^{2}+R^{2}\left(u_{1}^{2}+\rho^{2}\right)+2 R(1-R) u_{1}\right)^{1 / 2}\right) d \mu
$$

where

$$
d \mu=\frac{\left|(d-1) \mathbb{B}_{2}^{d-1}\right|}{\left|\mathbb{B}_{2}^{d}\right|} \rho^{d-2} d \rho d u_{1}
$$


defines a probability on $\mathbb{B}_{2}^{2} \cap\{\rho \geq 0\}$. Actually we get a probability $\mu_{d}$ for each dimension $d$, but since this is not relevant in the following argument we omit the reference to $d$ in the notation. Now from concavity and the fact that $u_{1}^{2}+\rho^{2} \leq 1$ we get

$$
I \leq 2 \int_{0}^{1} \int_{0}^{\left(1-u_{1}^{2}\right)^{1 / 2}} \omega\left(t\left((1-R)^{2}+R^{2}\left(u_{1}^{2}+\rho^{2}\right)\right)^{1 / 2}\right) d \mu \leq \omega\left(t\left((1-R)^{2}+R^{2}\right)^{1 / 2}\right) .
$$

Using $\inf _{1 / 2 \leq R \leq 1} \omega\left(t\left((1-R)^{2}+R^{2}\right)^{1 / 2}\right)=\omega\left(2^{-\frac{1}{2}} t\right)$ we obtain (3.14.4).

Regarding the assertion about the Hölder and Lipschitz classes, for $d>1$ the result follows from (3.14.4) with $\omega(t)=t^{\alpha}$. And for $d=1$, the upper bound is immediate from (3.14.18).

Remark 3.15. Consider the Lipschitz functions on $\mathbb{R}^{d}$. By the preceding theorem, for every $d$ we have $\|M\|_{\mathrm{Op}(1)} \leq 2^{-1 / 2}$ on $\operatorname{Lip}\left(\mathbb{R}^{d},\|\cdot\|_{2}\right)$. However, on $\operatorname{Lip}\left(\mathbb{R}^{d},\|\cdot\|_{\infty}\right),\|M\|_{\mathrm{Op}(1)}>$ $(d-1) /(d+1)$, by Corollary 3.4. Since $2^{-1 / 2}<5 / 7$, any optimal constant in the $\ell_{\infty}$ case, with $d \geq 6$, is strictly larger than all the optimal constants in the Euclidean case $(d=1,2,3, \ldots)$. This illustrates the fact that using different norms on $\mathbb{R}^{d}$ may result in obtaining very different best constants. But the opposite can also happen: Best constants are identical for the $\ell_{1}$ and $\ell_{\infty}$ norms, as we shall prove by showing that the corresponding integral formulas for $\|M\|_{\mathrm{Op}(\omega)}$ are actually the same.

Even though we are using different norms to define maximal operators and their associated moduli of continuity, we adopt the convention that the $s$-dimensional Hausdorff measure $\mathcal{H}^{s}$ is always defined via the Euclidean $\ell_{2}$ metric, and normalized by the factor $\pi^{s / 2} / \Gamma(1+s / 2)$, so if $s=d$ is a positive natural number, the cube of sidelength 1 has Hausdorff $d$ measure 1 . A consistent definition is required in order to use Fubini's Theorem, or more generally, the coarea formula. For example, by our convention the length of the polygonal curve $\mathbb{S}_{1}^{1}$ is $4 \sqrt{2}$, and not 8 , which would be obtained if length were computed using the $\ell_{1}$ distance instead of the Euclidean distance.

Theorem 3.16. Let $d \geq 2$, let $M$ be the uncentered maximal operator associated to balls defined by the $\ell_{1}$ norm, i.e., to cross-polytopes, and let $\omega$ be a modulus of continuity. Then (3.16.1)

$$
\|M\|_{\mathrm{Op}(\omega)}=\sup _{t>0} \inf _{1 / 2 \leq R \leq 1} \frac{d(d-1)}{2 \omega(t)} \int_{-1}^{1} \int_{0}^{1-\left|u_{1}\right|} \omega\left(t\left(\left|(1-R)+R u_{1}\right|+R \rho\right)\right) \rho^{d-2} d \rho d u_{1} .
$$

If we choose the same modulus $\omega$ in all dimensions, then we have $\lim _{d \rightarrow \infty}\|M\|_{\mathrm{Op}(\omega)}=1$.

Proof. Setting $c=(1-R) v$ in (2.14.1), we get

$$
\|M\|_{\mathrm{Op}(\omega)} \leq \sup _{t>0} \sup _{\left\{v \in \mathbb{R}^{d:}:\|v\|_{1}=1\right\}} \inf _{0<R<1} \frac{1}{\omega(t)} \int_{B(0,1)} \omega\left(t\|(1-R) v+R u\|_{1}\right) \frac{d u}{|B(0,1)|} .
$$

Optimality of $e_{1}$ in dimension 2 , follows from the fact that in this case cross-polytopes are just rotated squares, and for $d>2$, by Lemma 3.13. Thus

$$
\|M\|_{\mathrm{Op}(\omega)} \leq \sup _{t>0} \inf _{0<R<1} \frac{1}{\omega(t)} \int_{B(0,1)} \omega\left(t\left\|(1-R) e_{1}+R u\right\|_{1}\right) \frac{d u}{|B(0,1)|} .
$$


On the other hand, setting $v=e_{1}$ in (2.14.1) yields

$$
\|M\|_{\mathrm{Op}(\omega)} \geq \sup _{t>0} \inf _{\left\{c \in \mathbb{R}^{d}, 1>R>0:\left\|e_{1}-c\right\|_{1}=R\right\}} \frac{1}{\omega(t)} \int_{B(0,1)} \omega\left(t\|c+R u\|_{1}\right) \frac{d u}{|B(0,1)|} .
$$

It follows from Lemma 3.12 that, given $t$ and $R$, the infimum is attained in the last inequality when $c=(1-R) e_{1}$. Hence

$$
\|M\|_{\mathrm{Op}(\omega)} \geq \sup _{t>0} \inf _{0<R<1} \frac{1}{\omega(t)} \int_{\mathbb{B}_{1}^{d}} \omega\left(t\left\|(1-R) e_{1}+R u\right\|_{1}\right) \frac{d u}{|B(0,1)|},
$$

and thus we have equality.

Next, write $u=\left(u_{1}, y\right) \in \mathbb{R}^{d}$, and $y=\rho \eta$, where $\rho=\|y\|_{1}$ and $\|\eta\|_{1}=1$. By Fubini's Theorem

$$
\int_{\mathbb{B}_{1}^{d}} \omega\left(t\left\|(1-R) e_{1}+R u\right\|_{1}\right) d u=\int_{-1}^{1} \int_{\mathbb{B}_{1}^{d-1}\left(1-\left|u_{1}\right|\right)} \omega\left(t\left\|(1-R) e_{1}+R\left(u_{1}, y\right)\right\|_{1}\right) d y d u_{1} .
$$

Next we handle the vertical sections. As a reminder, we refer the reader to [Fe], pp. 248-250, or [EG], pp. 117-119, for basic information on the coarea formula

$$
\int_{\mathbb{R}^{d-1}} g(y)|J f(y)| d y=\int_{\mathbb{R}} \int_{\left\{f^{-1}(t)\right\}} g(y) d \mathcal{H}^{d-2}(y) d t .
$$

Let the Lipschitz function $f(y)$ be $\|y\|_{1}$. By definition, $|J f(y)|=\sqrt{\operatorname{det} d f(y) d f(y)^{t}}$, so for every $y$ with no coordinate equal to zero, a computation shows that $|J f(y)|=\sqrt{d-1}$. Thus, this is the case on almost all $\mathbb{R}^{d-1}$. Set $g(y)=1 /|J f(y)|$ and use (3.16.5) to obtain

$$
\begin{aligned}
\left|\mathbb{B}_{1}^{d-1}\right| & =\int_{\mathbb{B}_{1}^{d-1}} d y=\int_{0}^{1} \int_{\mathbb{S}_{1}^{d-2}(\rho)} \frac{1}{\sqrt{d-1}} d \mathcal{H}^{d-2}(y) d \rho= \\
& =\frac{\left|\mathbb{S}_{1}^{d-2}\right|}{\sqrt{d-1}} \int_{0}^{1} \rho^{d-2} d \rho=\frac{\left|\mathbb{S}_{1}^{d-2}\right|}{(d-1) \sqrt{d-1}} .
\end{aligned}
$$

From this equality and the coarea formula (once more), with

$$
g(y)=\frac{\omega\left(t\left(\left|(1-R)+R u_{1}\right|+R\|y\|_{1}\right)\right)}{|J f(y)|}
$$

we get

$$
\begin{gathered}
\int_{\mathbb{B}_{1}^{d-1}\left(1-\left|u_{1}\right|\right)} \omega\left(t\left\|(1-R) e_{1}+R\left(u_{1}, y\right)\right\|_{1}\right) d y \\
=\int_{0}^{1-\left|u_{1}\right|} \int_{\mathbb{S}_{1}^{d-2}(\rho)} \omega\left(t\left(\left|(1-R)+R u_{1}\right|+R \rho\right)\right) \frac{d \mathcal{H}^{d-2}(y)}{\sqrt{d-1}} d \rho \\
=\frac{1}{\sqrt{d-1}} \int_{0}^{1-\left|u_{1}\right|} \omega\left(t\left(\left|(1-R)+R u_{1}\right|+R \rho\right)\right) \int_{\mathbb{S}_{1}^{d-2}} \rho^{d-2} d \mathcal{H}^{d-2}(y) d \rho
\end{gathered}
$$




$$
=(d-1)\left|\mathbb{B}_{1}^{d-1}\right| \int_{0}^{1-\left|u_{1}\right|} \omega\left(t\left(\left|(1-R)+R u_{1}\right|+R \rho\right)\right) \rho^{d-2} d \rho .
$$

It is well known (and easy to compute) that $\left|\mathbb{B}_{1}^{d}\right|=\frac{2^{d}}{d !}$, so $\frac{\left|\mathbb{B}_{1}^{d-1}\right|}{\left|\mathbb{B}_{1}^{d}\right|}=\frac{d}{2}$ and we obtain

$$
\|M\|_{\mathrm{Op}(\omega)}=\sup _{t>0} \inf _{0 \leq R \leq 1} \frac{d(d-1)}{2 \omega(t)} \int_{-1}^{1} \int_{0}^{1-\left|u_{1}\right|} \omega\left(t\left(\left|(1-R)+R u_{1}\right|+R \rho\right)\right) \rho^{d-2} d \rho d u_{1} .
$$

To see that the infimum is attained when $R \in[1 / 2,1]$, we note that on $[0,1 / 2]$ the function $f_{1}(R)=1-R+R u_{1}+R \rho$ has a negative derivative for every $\rho \in\left[0,1-\left|u_{1}\right|\right)$. Finally, 1 is a uniform upper bound by Kinnunnen's Theorem, and concentration of measure near the vertical equator $\left\{u \in \mathbb{R}^{d}: u_{1}=0\right.$ and $\left.\|u\|_{1}=1\right\}$ entails that $\lim _{d \rightarrow \infty}\|M\|_{\mathrm{Op}(\omega)}=1$.

Observe, for instance, that unlike the case of cubes and the $\ell_{\infty}$ norm, it is not clear from formula (3.16.1) that $\|M\|_{\mathrm{Op}(\omega)}$ increases with the dimension. However, this must be the case, since given an arbitrary modulus, constants for the $\ell_{1}$ and the $\ell_{\infty}$ norms are equal in each dimension. Of course this is trivial for $d=1$, and clear for $d=2$, since in this case cross-polytopes are rotated squares. But when $d>2$ we have no justification to offer for this phenomenon, other than the proof below.

Theorem 3.17. Fix $d \geq 1$ and select a modulus of continuity $\omega$. Then $\|M\|_{\mathrm{Op}(\omega)}$ has exactly the same value regardless of whether distances and the maximal operator are computed according to the $\ell_{1}$ norm, or to the $\ell_{\infty}$ norm.

Recall that in the case of the $\ell_{\infty}$ norm the asymptotic value 1 was obtained by using concentration near the boundary of the cube $[-s, 1]^{d}$, and more precisely, near the norm 1 vectors, while in the $\ell_{1}$ case we utilized concentration near the vertical equator. By the preceding theorem one of these (different) arguments is redundant.

Proof. Fix $t>0$. To see that the values of $\|M\|_{\mathrm{Op}(\omega)}$ given by (3.1.1) and (3.16.1) are indeed the same, it is enough to show that

$$
\begin{aligned}
& \inf _{1 / 2 \leq R \leq 1} \frac{d(d-1)}{2} \int_{-1}^{1} \int_{0}^{1-\left|u_{1}\right|} \omega\left(t\left(\left|(1-R)+R u_{1}\right|+R \rho\right)\right) \rho^{d-2} d \rho d u_{1} \\
& \quad=\inf _{0 \leq s \leq 1} \frac{d}{(1+s)^{d}}\left(2^{d} \int_{0}^{s} z^{d-1} \omega(t z) d z+\int_{s}^{1}(z+s)^{d-1} \omega(t z) d z\right) .
\end{aligned}
$$

Write

$$
I:=\int_{-1}^{1} \int_{0}^{1-\left|u_{1}\right|} \omega\left(t\left(\left|(1-R)+R u_{1}\right|+R \rho\right)\right) \rho^{d-2} d \rho d u_{1}=I_{1}+I_{2}
$$

where

$$
I_{1}:=\int_{\frac{R-1}{R}}^{1} \int_{0}^{1-\left|u_{1}\right|} \omega\left(t\left((1-R)+R\left(u_{1}+\rho\right)\right)\right) \rho^{d-2} d \rho d u_{1}
$$


and

$$
I_{2}:=\int_{-1}^{\frac{R-1}{R}} \int_{0}^{1+u_{1}} \omega\left(t\left(R-1+R\left(\rho-u_{1}\right)\right)\right) \rho^{d-2} d \rho d u_{1} .
$$

To compute $I_{1}$, we use the change of variables $v=1-R+R\left(u_{1}+\rho\right), y=R u_{1}$ and Fubini's Theorem:

$$
\begin{gathered}
I_{1}=\int_{R-1}^{R} \int_{1-R+y}^{1-|y|+y} \omega(t v) \frac{(v+R-1-y)^{d-2}}{R^{d}} d v d y \\
=\frac{1}{R^{d}} \int_{0}^{1} \int_{\max \left\{R-1, \frac{v-1}{2}\right\}}^{v+R-1} \omega(t v)(v+R-1-y)^{d-2} d y d v \\
=\frac{1}{R^{d}(d-1)}\left(\int_{2 R-1}^{1} \omega(t v) \frac{(v+2 R-1)^{d-1}}{2^{d-1}} d v+\int_{0}^{2 R-1} \omega(t v) v^{d-1} d v\right) .
\end{gathered}
$$

Likewise, to compute $I_{2}$ we set $v=R-1+R\left(\rho-u_{1}\right), y=R u_{1}$, and interchange the order of integration:

$$
I_{2}=\int_{-R}^{R-1} \int_{R-1-y}^{2 R-1} \omega(t v) \frac{(v+1-R+y)^{d-2}}{R^{d}} d v d y=\frac{1}{R^{d}(d-1)} \int_{0}^{2 R-1} \omega(t v) v^{d-1} d v .
$$

Adding up we obtain

$$
I=\frac{1}{R^{d}(d-1)}\left(\int_{2 R-1}^{1} \omega(t v) \frac{(v+2 R-1)^{d-1}}{2^{d-1}} d v+2 \int_{0}^{2 R-1} \omega(t v) v^{d-1} d v\right) .
$$

To finish, set $s=2 R-1$, multiply by $d(d-1) / 2$, and take the corresponding infima to get (3.17.1).

We conclude this section with four questions and some variants of these. We conjecture that the following version of Theorems 3.14 and 3.16 holds for all $p \in(1,2)$ and $d \geq 2$ : For every modulus of continuity $\omega$ we have

$$
\begin{gathered}
\|M\|_{\mathrm{Op}(\omega)}=\sup _{t>0} \inf _{0 \leq R \leq 1} \frac{(d-1) \Gamma(1+d / p)}{2 \omega(t) \Gamma(1+1 / p) \Gamma(1+(d-1) / p)} \times \\
\int_{-1}^{1} \int_{0}^{\left(1-\left|u_{1}\right|^{p}\right)^{1 / p}} \omega\left(t\left(\left|(1-R)+R u_{1}\right|^{p}+R^{p} \rho^{p}\right)^{1 / p}\right) \rho^{d-2} d \rho d u_{1} .
\end{gathered}
$$

Let $q=p /(p-1)$ be the conjugate exponent of $p$. Choose the same modulus of continuity $\omega$ in every dimension $d$. Then

$$
\liminf _{d \rightarrow \infty}\|M\|_{\mathrm{Op}(\omega)} \geq \sup _{t>0}\left\{\frac{\omega\left(2^{-\frac{1}{q}} t\right)}{\omega(t)}\right\}
$$

and

$$
\limsup _{d \rightarrow \infty}\|M\|_{\mathrm{Op}(\omega)} \leq \inf _{r>1} \sup _{t>0}\left\{\frac{\omega\left(2^{-\frac{1}{q}} r t\right)}{\omega(t)}\right\}
$$


Suppose additionally that $\omega$ is concave. Then for every $d \geq 1$

$$
\|M\|_{\mathrm{Op}(\omega)} \leq \sup _{t>0}\left\{\frac{\omega\left(2^{-\frac{1}{q}} t\right)}{\omega(t)}\right\} .
$$

In particular, for the Hölder and Lipschitz classes we obtain

$$
\|M\|_{\mathrm{Op}(\alpha)} \leq 2^{-\frac{\alpha}{q}} \quad \text { for all } d \geq 1, \text { and } \quad \lim _{d \rightarrow \infty}\|M\|_{\mathrm{Op}(\omega)}=2^{-\frac{\alpha}{q}}
$$

The main obstacle for proving this result would be removed by a positive answer to the next question.

Question 1. Is $e_{1}$ a maximizing vector for $1<p<2$ when taking the supremum in (2.14.1)? Recall that by Lemma 3.13 it is enough to consider the case $d=2$.

It is plausible that best bounds increase with the dimension for $1 \leq p<\infty$, as it happens in the case $p=\infty$. This would immediately imply that the asymptotic bounds are also uniform upper bounds.

As we mentioned before, it seems likely that $v_{p}:=d^{-1 / p}(1,1, \ldots, 1)$ is an optimizing vector when $2<p<\infty$. But while using $e_{1}$ leads to simplification of the integral formulas, using $v_{p}$ does not. Furthermore, $e_{1}$ fits well with Fubini, in the sense that sections of $d$-balls yield $d-1$-balls if the sections are perpendicular to $e_{1}$. This does not happen with $v_{p}$, so a change of coordinates would not help on that respect. In any case, we suspect that on the range $2<p<\infty$, as $p \rightarrow \infty$ constants become increasingly worse and approach the bounds that hold for $p=\infty$.

Question 2. We have seen that if $f$ is Lipschitz and $M$ is the maximal function associated to euclidean balls, then for every dimension $d, \operatorname{Lip}(M f) \leq 2^{-1 / 2} \operatorname{Lip}(f)$, or equivalently, $\|D M f\|_{\infty} \leq 2^{-1 / 2}\|D f\|_{\infty}$. It is natural to expect a similar behavior for $p$ "close" to $\infty$. More precisely, given $c \in\left(2^{-1 / 2}, 1\right)$, is it possible to find a $p_{c}$ such that for all $p \geq p_{c}$, if $D f \in L^{p}$, then $\|D M f\|_{p} \leq c\|D f\|_{p}$ ? Or given $p>>1$, is it possible to find such a $c \in\left(2^{-1 / 2}, 1\right)$ ?

Current methods of proof use the $L^{p}$ inequalities satisfied by $M$ to obtain $W^{1, p}$ results. Since $\|M f\|_{p} \geq\|f\|_{p}$ always, this type of argument will never yield constants below 1 . On the other hand, if we fix $d$, by Corollary 2.13 we have $\|D M f\|_{\infty} \leq(1+d)^{-1} d\|D f\|_{\infty}$ for the maximal function associated to any ball, so it is natural to seek inequalities of the form $\|D M f\|_{p} \leq c_{p}\|D f\|_{p}$, where $c_{p}<1, p$ is high enough, and $M$ is defined via an arbitrary norm. At the other extreme, given $f \in W^{1,1}\left(\mathbb{R}^{d}\right)$ with $d \geq 2$, it is not known whether there is a constant $c_{1}$ (independent of $f$ ) such that $\|D M f\|_{1} \leq c_{1}\|f\|_{W^{1,1}\left(\mathbb{R}^{d}\right)}$. We mention that for some related maximal operators, such as, for instance, the strong maximal operator (where averages are taken over rectangles with sides parallel to the axes) such constants do not exist, cf. Theorem 2.21 of [AlPe2]. It follows from Theorem 2.5 of [AlPe that if $d=1$ then $\|D M f\|_{1} \leq c_{1}\|D f\|_{1}$, and $c_{1}=1$ is sharp. For $d \geq 2$ it is clear that if any such constant exists, it must be strictly larger that 1 (take $f$ to be radial, for instance). Whether or not $c_{1}<\infty$ for $d \geq 2$, one would expect $c_{p}>1$ for small values of $p, c_{p}<1$ for sufficiently large 
values of $p$, and $c_{p}=1$ for some "crossing" $p$, which will likely depend on $d$ and the ball used to define $M$. A motivation to obtain detailed information on $D M f$ comes not only from the possible use of $D M f$ as a substitute for $D f$, but also because it will yield new information about the maximal function itself.

On a more speculative mood, we note that where balls of arbitrarily small radii have to be taken into account, the function is "large" and the maximal function coincides with it, so maximal functions with smaller Lipschitz norms, and hence lower rate of decay "from the top", will tend to be larger in an $L^{p}$ sense. Since asymptotically constants are smaller for $\ell_{2}$ balls than for cubes or $\ell_{1}$ balls (and we believe this is also the case for other balls), it is tempting to conjecture that the maximal function associated to Euclidean balls is at least "as efficient" at capturing mass as maximal functions associated to other balls. Our results suggest this only in the weakest possible sense, since different norms are used to compute distances, the directions we consider when measuring the modulus of continuity are those of fastest decay and not some "average direction", and $L^{p}$ norms of extremal functions vary with $p$ (at least in the bounded case). Nevertheless, it seems worthwhile to try to find out whether the Euclidean maximal function, both in the centered and uncentered versions, has larger operator norm on $L^{p}$ spaces than maximal functions associated to other balls (needless to say, weak type results in this line would also be interesting). In fact, the weaker "comparison theorem" suggested next would already have many consequences.

Question 3. Let $\mathcal{M}_{e}$ denote the maximal operator, either centered or uncentered, associated to Euclidean balls, and $\mathcal{M}_{b}$ the corresponding (centered or uncentered) operator associated to some other ball (defined by a different norm). Prove or refute the following statement: For every $p \in(1, \infty)$,

$$
10^{78}\left\|\mathcal{M}_{e}\right\|_{L^{p}\left(\mathbb{R}^{d}\right) \rightarrow L^{p}\left(\mathbb{R}^{d}\right)} \geq\left\|\mathcal{M}_{b}\right\|_{L^{p}\left(\mathbb{R}^{d}\right) \rightarrow L^{p}\left(\mathbb{R}^{d}\right)} .
$$

Of course, $10^{78}$ is not important here, any other constant $c$ would do. But we do mean to emphasize that $10^{78}$ does not depend on anything, in particular not on $p$ or $d$. "Constants" that depend on the dimension are trivial to obtain by the equivalence of all norms in $\mathbb{R}^{d}$. The preceding conjecture, if true in the centered case, would imply that the uniform bounds in the dimension proved by E. M. Stein for Euclidean balls also hold for all other balls (including for instance, cubes) and all $p>1$. An opposing viewpoint can be found in [Mu], pg. 298, where it is suggested that this result may be false for cubes and $p \leq 3 / 2$.

The corresponding version of question 3 for the weak type $(1,1)$ constants is also interesting. An affirmative answer would entail that the best constants for the centered maximal function defined using euclidean balls diverge to infinity with the dimension, since this is the case for cubes, cf. [Al2]. Thus, a long standing open problem by Stein and Strömberg would be solved, cf. [StSt].

From the perspective of comparing the sizes of the maximal operators associated to euclidean balls and to cubes, it would probably be more telling if Lipschitz or Hölder constants were computed using the same distance (for example, the euclidean norm) in both cases. Of course, this "decoupling" between cubes and the $\ell_{2}$ norm means that we are outside the 
scope of Theorem 2.7. However, the maximal operator associated to cubes fits well with the product structure of $\mathbb{R}^{d}$, so it is natural, and common, to use it together with the natural distance on $\mathbb{R}^{d}$, the euclidean length. The next question seems therefore interesting to us:

Question 4. If in Theorem 3.1 we keep the maximal operator associated to cubes but consider the $\ell_{2}$ instead of the $\ell_{\infty}$ norm, how do the conclusions change?

\section{LOCAL RESULTS IN ONE DIMENSION.}

Next we study the local case in one dimension, that is, when the domain is a proper subinterval of $\mathbb{R}$. While the idea of the proof is the same as in the previous results, formally the next theorem does not follow from them, so we include the full argument. In fact, notation is considerably simplified by the fact that only intervals with $x$ as one endpoint need to be considered when computing $M f(x)$. In order to define any such interval it is enough to specify the other extreme, there is no need to talk about centers, radii and the relations between them.

We note that constants are worse in the local case than in the global case due, to the fact that a proper subinterval $I$ of $\mathbb{R}$ has at least one boundary point, say, for instance a left endpoint $a$. Then the decay restrictions imposed by the modulus of continuity only hold to the right of $a$, and so the level sets of an extremal function will be in general smaller than they would be if the function were defined over the whole real line. This is reflected in the different integral formulas; recall that for $\mathbb{R}$, Theorem 3.1 tells us that

$$
\omega(M f, t) \leq \min _{0 \leq s \leq 1} \frac{1}{1+s} \int_{-s}^{1} \omega(|f|, t u) d u .
$$

The existence of a boundary point entails that we must take $s=0$ in the local case, cf. (4.3.1) and (4.3.2) below.

Remark 4.1. If $f$ is uniformly continuous on a bounded interval $I$, then its modulus of continuity is constant on $[|I|, \infty)$, with value $\omega(f,|I|)$. Thus, if we are given a modulus $\omega$, and $|I|<\infty$, we cannot expect to find a function $\psi \in \operatorname{Lip}(\omega, I)$ with $\omega(\psi, t)=\omega(t)$ for all $t>0$. In general, the best we can do is to find $\psi$ so that $\omega(\psi, t)=\omega(t)$ on $(0,|I|]$.

Remark 4.2. By an interval $I$ we always mean a nondegenerate interval, so the empty set $(a, a]$ and points $[a, a]$ are excluded. Also, the requirement that subintervals be proper leaves out the already studied case $I=\mathbb{R}$. Other than that, there are no restrictions on the subintervals of $\mathbb{R}$ considered in Theorem 4.3. Nevertheless, for convenience we shall assume in the proof that intervals are not open. The open case is handled in essentially the same way, via a limit argument. So $I$ will contain at least one endpoint. To simplify notation, we shall assume that this endpoint is the origin, and furthermore, that it is the left endpoint of $I$. 
Theorem 4.3. Let $I \subset \mathbb{R}$ be a proper subinterval, and let $f: I \rightarrow \mathbb{R}$ be locally integrable. Then, for every $t>0$,

$$
\omega(M f, t) \leq \int_{0}^{1} \omega(|f|, t u) d u .
$$

Inequality (4.3.1) is sharp in the sense that for every modulus of continuity $\omega$ and every $\delta>0$ with $\delta \leq|I|$, there exists a nonnegative, uniformly continuous function $\psi: I \rightarrow \mathbb{R}$ such that for all $t \in(0, \delta)$, we have $\omega(\psi, t)=\omega(t)$ and

$$
\omega(M \psi, t)=\int_{0}^{1} \omega(t u) d u .
$$

Moreover, if $\omega$ is bounded, then the function $\psi$ can be chosen so that it satisfies $\omega(\psi, t)=\omega(t)$ and (4.3.2) for all $t>0$ with $t<|I|$.

Proof. Assume $f \geq 0$, and note that when we evaluate $M f(x)$, taking the supremum over all intervals containing $x$ yields the same value as taking the supremum over intervals having $x$ as a boundary point (in other words, $M f$ is the maximum of the right and left one sided maximal functions). Let $x, y \in I$ be such that $M f(y)<M f(x)$. Then

$$
\begin{gathered}
M f(x)-M f(y)=\sup _{S \in I} \frac{1}{S-x} \int_{x}^{S} f(u) d u-M f(y) \\
\leq \sup _{S \in I}\left(\frac{1}{S-x} \int_{x}^{S} f(u) d u-\frac{1}{S-y} \int_{y}^{S} f(u) d u\right) \\
\leq \sup _{S \in I} \int_{0}^{1}|f(x+(S-x) u)-f(y+(S-y) u)| d u \leq \int_{0}^{1} \omega(|f|,|x-y| u) d u .
\end{gathered}
$$

Now using a symmetry argument between $x$ and $y$, and taking the supremum over $|x-y| \leq t$, (4.3.1) follows.

We prove the optimality of (4.3.1) on the interval $I=[0, \infty)$. The argument can be easily adapted to other proper subintervals of $\mathbb{R}$. If $\omega$ is bounded, set $\psi:=\|\omega\|_{L^{\infty}([0, \infty))}-\omega$. It is immediate from the definitions that $\omega(\psi, t)=\omega(t)$. Since $\psi$ is decreasing, $M \psi(t)=$ $t^{-1} \int_{0}^{t} \psi(u) d u=\int_{0}^{1} \psi(t u) d u$, so

$$
\omega(M \psi, t) \geq M \psi(0)-M \psi(t)=\|\omega\|_{L^{\infty}([0, \infty))}-\int_{0}^{1} \psi(t u) d u=\int_{0}^{1} \omega(t u) d u .
$$

Thus, by (4.3.1) we have equality. If $\omega$ is unbounded, the result follows from the previous case by fixing $\delta>0$ and considering the bounded modulus $\omega_{\delta}(t):=\min \{\omega(t), \omega(\delta)\}$.

Remark 4.4. In (4.3.4) we are crucially using that $t<|I|$. Suppose $I=[0,1]$, and let $\omega(t)=\omega(\psi, t)=\min \{t, 1\}$, where $\psi(x)=1-x$. Then for all $t \geq 1, \omega(M \psi, t)=1 / 2<$ $1=\lim _{t \rightarrow \infty} \int_{0}^{1} \omega(t u) d u$. For the same reason, the restriction $t<|I|$ is also needed in (4.5.1) below. 
Recall that $\|f\|_{\operatorname{Lip}(\omega, I)}=\sup _{t>0} \omega(f, t) / \omega(t)$. In the next result we use the following notation: $\psi_{T}:[0, \infty) \rightarrow[0, \infty)$ is defined by $\psi_{T}:=(T-\omega)^{+}$. We shall see that only the functions $\psi_{T}$ need to be taken into account when computing $\|M\|_{\mathrm{Op}(\omega)}$. If $\omega$ is bounded we set $T=\|\omega\|_{L^{\infty}([0, \infty))}$ (as usual) and just write $\psi$.

Corollary 4.5. Let $\omega$ be a modulus of continuity and let I be a proper subinterval of $\mathbb{R}$. On $\operatorname{Lip}(\omega, I)$,

$$
\|M\|_{\mathrm{Op}(\omega)}=\sup _{0<t<|I|} \frac{1}{\omega(t)} \int_{0}^{1} \omega(t u) d u .
$$

In the special case where $\omega(t)=t^{\alpha}, \alpha \in(0,1]$, that is, over the Hölder and Lipschitz classes,

$$
\|M\|_{\mathrm{Op}(\alpha)}=\frac{1}{1+\alpha} .
$$

Proof. If $\omega$ is bounded, (4.5.1) follows immediately from the fact that there is an extremal function $\psi$ such that for all $t>0$ with $t<|I|, \omega(\psi, t)=\omega(t)$ and $\omega(M \psi, t)=\int_{0}^{1} \omega(t u) d u$. And if $|I|<\infty$, replacing $\omega$ by $\omega^{\prime}:=\min \{\omega, \omega(|I|)\}$, we are back to the bounded case.

Suppose next that both $\omega$ and $I$ are unbounded. Without loss of generality we may assume, in order to simplify notation, that $I=[0, \infty)$. Select for each $n \geq 1$ a norm one function $f_{n} \in \operatorname{Lip}(\omega, I)$ approximating the operator norm of $M$ to within $1 / n$. Then

$$
\|M\|_{\mathrm{Op}(\omega)} \leq\left\|M f_{n}\right\|_{\operatorname{Lip}(\omega)}+\frac{1}{n} \leq \sup _{t>0} \frac{1}{\omega(t)} \int_{0}^{1} \omega\left(\left|f_{n}\right|, t u\right) d u+\frac{1}{n} \leq \sup _{t>0} \frac{1}{\omega(t)} \int_{0}^{1} \omega(t u) d u+\frac{1}{n} .
$$

On the other hand, since each $\psi_{T}$ has norm 1 ,

$$
\begin{gathered}
\|M\|_{\mathrm{Op}(\omega)} \geq \sup _{T>0}\left\|M \psi_{T}\right\|_{\operatorname{Lip}(\omega)}=\sup _{T>0}\left\{\sup _{0<t<T} \frac{1}{\omega(t)} \int_{0}^{1} \omega\left(\psi_{T}, t u\right) d u\right\} \\
=\sup _{T>0}\left\{\sup _{0<t<T} \frac{1}{\omega(t)} \int_{0}^{1} \omega(t u) d u\right\}=\sup _{t>0} \frac{1}{\omega(t)} \int_{0}^{1} \omega(t u) d u .
\end{gathered}
$$

If $\omega(t)=t^{\alpha}, \alpha \in(0,1]$, then

$$
\|M\|_{\mathrm{Op}(\omega)}=\sup _{0<t<|I|} \frac{1}{\omega(t)} \int_{0}^{1} \omega(t u) d u=\int_{0}^{1} u^{\alpha} d u=\frac{1}{1+\alpha} .
$$

Remark 4.6. The sharp bounds given above yield information about related inequalities. For instance, in Theorem 5.1 of [AlPe the following Landau type inequality is proven: If $u:[0, \infty) \rightarrow \mathbb{R}$ is an absolutely continuous function such that its derivative is of bounded variation, then

$$
\left\|u^{\prime}\right\|_{\infty}^{2} \leq 48\|u\|_{\infty}\left(\left\|D M\left(u^{\prime+}\right)\right\|_{\infty}+\left\|D M\left(u^{\prime-}\right)\right\|_{\infty}\right)
$$


It is natural to seek a lower bound on the best possible constant $c$ that can replace 48 in (4.6.1). Recall that the classical (sharp) Landau inequality assumes more regularity on the part of $u^{\prime}$ : If it is absolutely continuous on $[0, \infty)$, then

$$
\left\|u^{\prime}\right\|_{\infty}^{2} \leq 4\|u\|_{\infty}\left\|u^{\prime \prime}\right\|_{\infty}
$$

As noted in Remark 5.5, of AlPe, (4.6.1) implies Landau's inequality, save for the issue of best constants. Using Corollary 4.5 and (4.6.1) we have that $\left\|D M\left(u^{\prime+}\right)\right\|_{\infty}+\left\|D M\left(u^{\prime-}\right)\right\|_{\infty} \leq$ $2^{-1}\left\|D u^{\prime+}\right\|_{\infty}+2^{-1}\left\|D u^{\prime-}\right\|_{\infty} \leq\left\|D u^{\prime}\right\|_{\infty}$, so $4 \leq c \leq 48$. On the whole real line, the constant from Theorem 5.1 of $\mathrm{AlPe}$ appearing in (4.6.1) is 24 instead of 48, and the best constant in the sharp Landau's inequality (4.6.2) is 2 instead of 4. Arguing as before and using Corollary [3.6, we have $\left\|D M\left(u^{\prime+}\right)\right\|_{\infty}+\left\|D M\left(u^{\prime-}\right)\right\|_{\infty} \leq 2(\sqrt{2}-1)\left\|D u^{\prime}\right\|_{\infty}$, so $1 /(\sqrt{2}-1) \leq c \leq 24$. Thus, on $\mathbb{R}$ the best constant appearing in the generalized Landau inequality (4.6.1) is strictly larger than the best constant 2 in the classical Landau inequality.

Remark 4.7. By Corollary 4.5 the functions $\psi_{\alpha}(x):=\max \left\{1-x^{\alpha}, 0\right\}$ are extremal in the classes $\operatorname{Lip}(\alpha)([0, \infty))$. Now $M \psi_{\alpha}(x)=1-\frac{x^{\alpha}}{1+\alpha}$ on $0 \leq x<1$ (and $M \psi_{\alpha}(x)=\frac{\alpha}{(1+\alpha) x}$ on $1 \leq x<\infty)$. Thus, the Hölder or Lipschitz exponent of $M \psi_{\alpha}$ is no better than that of $\psi_{\alpha}$. But in this example there is only one "bad point": For every $\varepsilon>0$, both $\psi_{\alpha}$ and $M \psi_{\alpha}$ are Lipschitz on $[\varepsilon, \infty)$. In view of the regularizing properties of $M$, one may wonder, for instance, whether $M f$ must have a better Hölder exponent than $f$ save for small sets, or in some "almost everywhere" sense. We shall see below that the answer is negative.

We also note that the preservation of regularity does not extend to the $C^{r}$ classes. To see this, let $f$ be the sum of two smooth bump functions with disjoint supports and recall that $M f$ is the maximum of the right and left one sided maximal functions. This entails that between the bumps of $f, M f$ achieves its minimum value at a point of non-differentiability, since the right and left derivatives of $M f$ are nonzero there and have opposite signs.

Next we show that a Hölder condition on $f$ is not sufficient to ensure the differentiability almost everywhere of $M f$, so in particular $M f$ can fail to be absolutely continuous. The following example also shows that the Hölder exponent of $M f$ may be as bad as that of $f$ on a large set. To prove this we suitably modify the fat Cantor set defined in Example 4.2 of AlPe, and the functions used there.

Example 4.8. For every $\alpha \in(0,1)$ there exists a function $f \in \operatorname{Lip}_{\alpha}([0,1])$, with $\operatorname{Lip}_{\alpha}(f)=1$, such that $M f$ is not differentiable on a set $E$ of positive measure. Furthermore, given any $\beta \in(\alpha, 1), M f$ is not locally Hölder $(\beta)$ at any point of $E$. More precisely, given any $x \in E$, and any interval $I$, relatively open in $[0,1]$ and with $x \in I$, we have $M f \notin \operatorname{Lip}_{\beta}(I)$.

Proof: As in Example 4.2 of [AlPe], and unlike the usual construction of the Cantor set, instead of removing the "central part" of every interval at each stage, we remove several parts. Let $F_{0}=[0,1]$ and let $F_{n}$ be the finite union of closed subintervals of $[0,1]$ obtained at step $n$ of the construction, to be described below. As usual $C:=\cap_{n} F_{n}$. Denote by $\ell(J)$ and $r(J)$ the left and right endpoints of an interval $J$. At stage $n$ we remove $2^{-2 n}$ of the mass in the 
preceding set, so $|C|>1-\left|\cup_{1}^{\infty} F_{n}^{c}\right| \geq 1-\sum_{1}^{\infty} 2^{-2 n}=2 / 3$. Let $I_{n-1}$ be a component of $F_{n-1}$, and let $O(n, x)$ the open interval centered at $x$ of length $2^{-4 n}\left|I_{n-1}\right|$. Select the unique $2^{2 n}$ points $x_{1}, x_{2} \ldots, x_{2^{2 n}} \in I_{n-1}$ such that i) $\ell\left(I_{n-1}\right)<x_{1}<\cdots<x_{2^{2 n}}<r\left(I_{n-1}\right)$, and ii) after the removal of the $2^{2 n}$ open intervals $O\left(n, x_{i}\right)$ from $I_{n-1}$, the $2^{2 n}+1$ remaining components have equal length, which therefore must be $\left(1-2^{-2 n}\right)\left|I_{n-1}\right| /\left(2^{2 n}+1\right)$. Thus, $x_{2}-x_{1}=x_{i+1}-x_{i}$ for $i<2^{2 n}$, while $r\left(I_{n-1}\right)-x_{2^{2 n}}=x_{1}-\ell\left(I_{n-1}\right)<x_{2}-x_{1}$. Note that the extremes of $I_{n-1}$ are left untouched.

Repeat the same process with the other components of $F_{n-1}$ to obtain $F_{n}$, which by construction is a disjoint union of closed intervals, all of length $\left(1-2^{-2 n}\right)\left|I_{n-1}\right| /\left(2^{2 n}+1\right)$.

Next we define the function $f$. Given $t>0$, set $g_{t}(x)=1-(t-x)^{\alpha}$ on $[0, t)$ and extend it to $(-t, t)$ as an even function. Then let $f$ be identically 1 on $C$, and let $f(x):=g_{t}(x-s)$ on each interval of type $O(j, s)$, where $t$ is half the length of $O(j, s)$. To see why $f \in \operatorname{Lip}_{\alpha}([0,1])$ and $\operatorname{Lip}_{\alpha}(f)=1$, note that if $x \in C$ and $y \in[0,1] \backslash C$, say, with $y<x$, then there is a unique $O(n, w)$ containing $y$, so we can replace $x$ by $r(O(n, w))$ and then $f(x)-f(y)=$ $f(r(O(n, w)))-f(y)$, while $r(O(n, w))-y \leq x-y$; the case where $x$ and $y$ belong to different components of $[0,1] \backslash C$ can be reduced to the previous one: Let $y \in O(n, u), x \in O(m, w)$, and suppose $y<x$. If $f(x)<f(y)$, replace $y$ with $\ell(O(m, w))$; otherwise, replace $x$ with $r(O(n, u))$. So it is enough to verify that $f$ has the desired properties on the closure of an arbitrary $O(j, s)$, and this is true since it holds for $|x|^{\alpha}$, its translates, reflections, etc. Next, let $f_{n}:=1$ on $F_{n}$ and $f_{n}:=f$ on $[0,1] \backslash F_{n}$. Then $f \leq f_{n}$, so $M f \leq M f_{n}$, and of course the latter function is easier to work with. Let $x_{i}$ and $O\left(n, x_{i}\right)$ be as above. For notational convenience, suppose we are taking $i=2$; the same reasoning applies to the other $x_{j}$. To evaluate $M f_{n}\left(x_{2}\right)$ we only need to consider intervals $J$ that have $x_{2}$ as an endpoint and, we claim, omit both $x_{1}$ and $x_{3}$. To see this, assume that $x_{2}=\ell(J)$. Since $f_{n}\left(x_{2}\right)<M f_{n}\left(x_{2}\right)<1$, an optimal $J$ must satisfy $f_{n}(r(J))=M f_{n}\left(x_{2}\right)$. Otherwise we could improve the average by either shortening or enlarging $J$. Thus, $r(J) \in O\left(n, x_{3}\right) \cap\left\{x<x_{3}\right\}$. Of course, the situation is completely symmetrical if $x_{2}=r(J)$ (here $\ell(J) \in O\left(n, x_{1}\right) \cap\left\{x>x_{1}\right\}$ ), so we keep the assumption $x_{2}=\ell(J)$. Next, define $h \geq f_{n}$ by setting $h \equiv 1$ on $O\left(n, x_{3}\right) \cap\left\{x<x_{3}\right\}$ and $h \equiv f_{n}$ elsewhere. Clearly

$$
\begin{gathered}
M f_{n}\left(x_{2}\right)<\frac{1}{x_{3}-x_{2}} \int_{x_{2}}^{x_{3}} h=\frac{\left(\frac{1-2^{-2 n}}{2^{2 n}+1}+2^{-4 n-1}\right)\left|I_{n-1}\right|+\int_{0}^{2^{-4 n-1}\left|I_{n-1}\right|} 1-x^{\alpha} d x}{\left(\frac{1-2^{-2 n}}{2^{2 n}+1}+2^{-4 n}\right)\left|I_{n-1}\right|} \\
=1-\frac{2^{(-4 n-1)(\alpha+1)}}{\left(\frac{1-2^{-2 n}}{2^{2 n}+1}+2^{-4 n}\right)(\alpha+1)}\left|I_{n-1}\right|^{\alpha}<1-2^{-4 \alpha n-2 n-\alpha-2}\left|I_{n-1}\right|^{\alpha} .
\end{gathered}
$$

Fix $z \in C \cap\{M f=1\}$. For each $n$, let $I_{n, z}$ be the component of $F_{n}$ that contains $z$, and let $w_{n}$ be midpoint of the nearest interval $O(n)$ deleted at step $n$ (if there are two such midpoints, pick any). Then $\left|z-w_{n}\right|<2^{-2 n}\left|I_{n-1}\right|$, and furthermore $\left|I_{n-1}\right|<2^{-n(n-1)}$, since the number of components of the set $F_{n-1}$ is $\prod_{i=1}^{n-1}\left(2^{2 i}+1\right)>\prod_{i=1}^{n-1} 2^{2 i}=2^{n(n-1)}$. Thus, for every $\beta \in(\alpha, 1]$ 
we have

$$
\limsup _{w \rightarrow z} \frac{|M f(z)-M f(w)|}{|z-w|^{\beta}} \geq \limsup _{n \rightarrow \infty} \frac{1-M f_{n}\left(w_{n}\right)}{\left|z-w_{n}\right|^{\beta}} \geq \lim _{n \rightarrow \infty} \frac{2^{-4 \alpha n-2 n-\alpha-2}}{2^{-2 \beta n}\left(2^{-n(n-1)}\right)^{\beta-\alpha}}=\infty .
$$

Since $M f \geq f$ a.e., the sets $E:=C \cap\{M f=1\}$ and $C$ have the same measure, so taking $\beta=1$ it follows that $M f$ is not differentiable on a set of measure at least $2 / 3$, while for $\beta \in(\alpha, 1)$ we obtain the failure of the local Hölder condition with exponent $\beta$.

Remark 4.9. The following example shows that local, higher dimensional generalizations of the preceding results may fail. Recall that in Definition 2.1 the balls in $\mathbb{R}^{d}$ we average over, are assumed to be fully contained in the (open) domain $U$ of $f$. Let $U=$ $\left\{(x, y): x \in \mathbb{R}^{d}, y \in \mathbb{R}, y>\exp (-1 /|x|)\right\}$ be a domain with a cusp and let $f(x, y)=y$. Then $\lim _{y \downarrow 0} M f(0, y)=0$, while $M f(0,1)=\infty$.

\section{REFERENCES}

[Al1] Aldaz, J. M. Remarks on the Hardy-Littlewood maximal function. Proceedings of the Royal Society of Edinburgh A, 128 (1998), 1-9.

[A12] Aldaz, J. M. The weak type $(1,1)$ bounds for the maximal function associated to cubes grow to infinity with the dimension. To appear in Ann. Math. Available at the Mathematics ArXiv.

[Al3] Aldaz, J. M. Dimension dependency of the weak type $(1,1)$ bounds for maximal functions associated to finite radial measures. Bull. Lond. Math. Soc. 39 (2007), no. 2, 203-208. Also available at the Mathematics ArXiv.

[AlPe] Aldaz, J. M.; Pérez Lázaro, J. Functions of bounded variation, the derivative of the one dimensional maximal function, and applications to inequalities. Trans. Amer. Math. Soc. 359 (2007) no. 5, 24432461. Also available at the Mathematics ArXiv.

[AlPe2] Aldaz, J. M.; Pérez Lázaro, J. Boundedness and unboundedness results for some maximal operators on functions of bounded variation. J. Math. An. Appl. Volume 337 (2008) Issue 1, 130-143. Also available at the Mathematics ArXiv.

[AlPe3] Aldaz, J. M.; Pérez Lázaro, J. Regularity of the Hardy-Littlewood maximal operator on block decreasing functions. Studia Math. Volume 194 (2009) 253-277. Also available at the Mathematics ArXiv.

[AlPe4] Aldaz, J. M.; Pérez Lázaro, J. The best constant for the centered maximal operator on radial functions.. To appear in Math. Ineq. Appl. Available at the Mathematics ArXiv.

[AlPe5] Aldaz, J. M.; Pérez Lázaro, J. Behavior of weak type bounds for high dimensional maximal operators defined by certain radial measures. To appear in Positivity, DOI: 10.1007/s11117-010-0051-2. Available at the Mathematics ArXiv.

[An] Anderson, T. W. The integral of a symmetric unimodal function over a symmetric convex set and some probability inequalities. Proc. Amer. Math. Soc. 6, (1955). 170-176.

[BD] Blackwell, D.; Dubins, L. E. Sharp bounds on the distribution of the Hardy-Littlewood maximal function. Proc. Amer. Math. Soc. 14 (1963) 450-453.

[Bou1] Bourgain, J. On high-dimensional maximal functions associated to convex bodies. Amer. J. Math. 108 (1986), no. 6, 1467-1476.

[Bou2] Bourgain, J. On the $L^{p}$-bounds for maximal functions associated to convex bodies in $R^{n}$. Israel J. Math. 54 (1986), no. 3, 257-265.

[Bou3] Bourgain, J. On dimension free maximal inequalities for convex symmetric bodies in $R^{n}$. Geometrical aspects of functional analysis (1985/86), Lecture Notes in Math. 1267 (Springer, Berlin, 1987), 168176. 
[Ca] Carbery, A. An almost-orthogonality principle with applications to maximal functions associated to convex bodies. Bull. Amer. Math. Soc. (N.S.) 14 (1986), no. 2, 269-273.

[CF] Capri, O. N.; Fava, N. A. Strong differentiability with respect to product measures Studia Math. (1984) 173-178.

[CLM] Colzani, L.; Laeng, E.; Morpurgo, C. Symmetrization and norm of the Hardy-Littlewood maximal operator on Lorentz and Marcinkiewicz spaces. J. London Math. Soc., 2008 77(2):349-362; doi:10.1112/jlms/jdm111

[EG] Evans, Lawrence C.; Gariepy, Ronald F. Measure theory and fine properties of functions. Studies in Advanced Mathematics. CRC Press, Boca Raton, FL, 1992. viii+268 pp. ISBN: 0-8493-7157-0

[Fe] Federer, Herbert. Geometric measure theory. Die Grundlehren der mathematischen Wissenschaften, Band 153 Springer-Verlag New York Inc., New York 1969 xiv+676 pp.

[Ga] Gardner, R. J. The Brunn-Minkowski inequality. Bull. Amer. Math. Soc. (N.S.) 39 (2002), no. 3, 355-405.

[GK] Grafakos, L.; Kinnunen, J. Sharp inequalities for maximal functions associated with general measures. Proc. Roy. Soc. Edinburgh Sect. A 128 (1998), no. 4, 717-723.

[GM] Grafakos, L.; Montgomery-Smith, S. Best constants for uncentred maximal functions. Bull. London Math. Soc. 29 (1997), no. 1, 60-64.

[GMM] Grafakos, L.; Montgomery-Smith, S.; Motrunich, O. A sharp estimate for the Hardy-Littlewood maximal function. Studia Math. 134 (1999), no. 1, 57-67.

[HaOn] Hajłasz, Piotr; Onninen, Jani. On boundedness of maximal functions in Sobolev spaces. Ann. Acad. Sci. Fenn. Math. 29 (2004), no. 1, 167-176.

[Ki] Kinnunen, J. The Hardy-Littlewood maximal function of a Sobolev function. Israel J. Math. 100 (1997), 117-124.

[KiLi] Kinnunen, J.; Lindqvist, P. The derivative of the maximal function. J. Reine Angew. Math. 503 (1998), 161-167.

[KiSa] Kinnunen, J.; Saksman, E. Regularity of the fractional maximal function. Bull. London Math. Soc. 34 (2003),no. 4, 529-535.

[Lu] Luiro, H. Continuity of the maximal operator in Sobolev spaces. Proc. Amer. Math. Soc. 135 (2007), no. $1,243-251$.

[Me1] Melas, A. D. On the centered Hardy-Littlewood maximal operator. Trans. Amer. Math. Soc. 354 (2002), no. 8, 3263-3273.

[Me2] Melas, A. D. The best constant for the centered Hardy-Littlewood maximal inequality. Ann. of Math. (2) 157 (2003), no. 2, 647-688.

[Mu Müller, D. A geometric bound for maximal functions associated to convex bodies. Pacific J. Math. 142 (1990), no. 2, 297-312.

[NaTa] Naor, Assaf; Tao, Terence. Random Martingales and localization of maximal inequalities. J. Func. An. 259 (2010) Issue 3, 731-779. Available at the Mathematics ArXiv.

[St1] Stein, E. M. The development of square functions in the work of A. Zygmund. Bull. Amer. Math. Soc. (N.S.) 7 (1982), no. 2, 359-376.

[St2] Stein, E. M. Three variations on the theme of maximal functions. Recent progress in Fourier analysis (El Escorial, 1983), (North-Holland Math. Stud., 111, North-Holland, Amsterdam, 1985), 229-244.

[St3] Stein, E. M. Harmonic analysis: real-variable methods, orthogonality, and oscillatory integrals. With the assistance of Timothy S. Murphy. (Princeton Mathematical Series, 43. Monographs in Harmonic Analysis, III. Princeton University Press, Princeton, NJ, 1993).

[StSt] Stein, E. M.; Strömberg, J. O. Behavior of maximal functions in $R^{n}$ for large $n$. Ark. Mat. 21 (1983), no. 2, 259-269.

[Ta] Tanaka, H. A remark on the derivative of the one-dimensional Hardy-Littlewood maximal function. Bull. Austral. Math. Soc. 65 (2002) no. 2, 253-258. 
[Web] Webster, R. J. Convexity. Oxford University Press (1997).

Departamento de Matemáticas, Universidad Autónoma de Madrid, 28049 Madrid, Spain. E-mail address: jesus.munarriz@uam.es

Dipartimento di Matematica, Università di Milano-Bicocca, Edificio U5, via R. Cozzi 53, 20125 Milano, Italia.

E-mail address: leonardo.colzani@unimib.it

Departamento de Matemáticas y Computación, Universidad de La Rioja, 26004 Logroño, LA Rioja, SpAin.

E-mail address: javier.perezl@unirioja.es 